\title{
Three-dimensional map for a piecewise-linear capsule system with bidirectional drifts
}

\author{
Bingyong Guo, Yang Liu* \\ College of Engineering, Mathematics and Physical Sciences, University of Exeter, North Park Road, Exeter, EX4 4QF, UK
}

\section{H I G H L I G H T S}

- This paper studies a three-dimensional map for a piecewise-linear capsule system.

- The capsule system has two degrees-of-freedom involving dry friction and impact.

- Global and local dynamics of the system are studied by using the proposed map.

- All possible local mappings are obtained for a wide range of parameter variation.

- The map can be used to explain the occurrence of different bifurcations.

\section{A R T I C L E I N F O}

\section{Article history:}

Received 21 January 2019

Received in revised form 30 March 2019

Accepted 23 April 2019

Available online $\mathrm{xxxx}$

Communicated by G. Stepan

\section{Keywords:}

Vibro-impact

Non-smooth dynamical system

Bifurcation

Dry friction

Discrete mapping

\begin{abstract}
A B S T R A C T
A three-dimensional map is studied in this paper to provide a fundamental understanding for the vibroimpact capsule system, which is a non-autonomous two degrees-of-freedom non-smooth dynamical system consisting of soft impact and dry friction with forward and backward drifts. By using the map, one can investigate the global and local dynamics of the system and construct all possible local mappings for a wide range of parameter variation. An example study by varying the amplitude of external excitation of the system is presented. Our results show that the proposed map can effectively explain the occurrence of the boundary-intersection crossing and the sliding bifurcations observed in the system.
\end{abstract}

(C) 2019 Elsevier B.V. All rights reserved.

\section{Introduction}

Locomotion mechanism has been widely used in engineering and healthcare, such as oil/gas pipe inspection [1], disaster rescue [2], and medical diagnosis and treatment [3]. These devices have external moving parts, e.g., wheels, legs or paddles, which are not suitable for the applications in complex environments, e.g., in the gastrointestinal tract. On the other hand, a locomotion mechanism without any external moving parts may have many advantages in these applications. For example, the twomass system [4] shown in Fig. 1(a), which consists of a movable mass within a shell, has simpler and more cost-effective design than the legged capsule [5]. By controlling its inner mass periodically [6], the entire system can move forward or backward when the interaction force applied on the shell exceeds its environmental resistance. Inspired by the drifting oscillator [7], a two degrees-of-freedom vibro-impact capsule system with bidirectional drifts [8], shown in Fig. 1(b), can provide effective locomotion in a confined environment. The system consists of an

\footnotetext{
* Corresponding author.

E-mail address: y.liu2@exeter.ac.uk (Y. Liu).
}

inner mass attached to a capsule via a spring and a damper at one end. A secondary spring is attached to the other end of the capsule to provide visco-elastic impacts when the relative displacement between the inner mass and the secondary spring is greater than or equal to their gap. As the inner mass is driven by a harmonic force, the interaction force can overcome capsule-surface resistance and drive the capsule to move forward or backward as a whole. In order to optimise its trajectory for directional control and energy saving, understanding the dynamics of such a system, e.g., prediction of bifurcation, is vital.

Our previous work on the capsule system focused on its modelling [8], motion control [9] and model verification [10]. Both numerical and experimental studies indicate the existence of optimal orbits of the capsule system to achieve the best progression or the lowest energy consumption, and a position feedback control method was developed to follow these orbits. The capsule system takes the use of non-smoothness of the system, i.e., friction and impacts, to achieve desired locomotion. To analyse its dynamics, the system can be modelled as a piecewise-linear drifting oscillator with a set of linear and smooth subsystems, whose subspaces are divided by the non-smooth boundaries induced by friction and impacts. Although local dynamics of the 


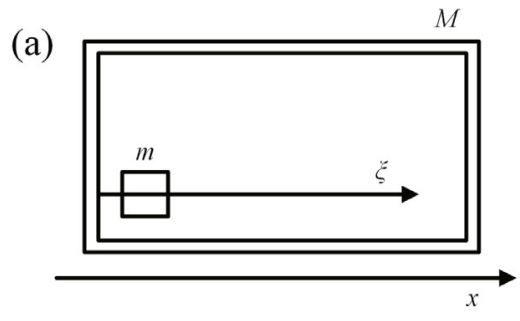

(b)

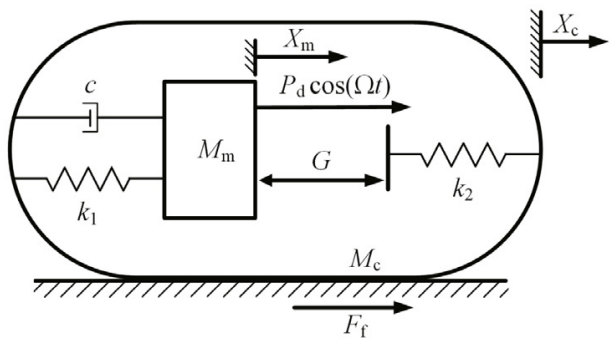

Fig. 1. Physical models of (a) the two-mass system [4], and (b) the vibro-impact capsule system with bidirectional drifts.

system has been extensively studied, e.g., [8,9,11], the root causes of bifurcation and change of locomotion direction are still not fully understood. Therefore, the global map which can interpret the switching mechanisms of the capsule dynamics across nonsmooth boundaries is required. In addition, the capsule system is sensitive to its system and control parameters, e.g., the frequency and amplitude of excitation, and any parameter variation may cause a qualitative change of its dynamics. However, there is no systematic approach to study such a complex dynamics, which is the rationale of this paper.

This paper aims to study a three-dimensional (3D) map for the vibro-impact capsule system, to depict its global and local dynamics in the presence of non-smooth boundaries. Discrete mapping approaches have been widely used to investigate dynamical systems subjected to non-smoothness, e.g., [12-17]. Shaw and Holmes [12] first adopted a discrete mapping approach to study the dynamics of a periodically forced piecewise-linear oscillator. Nordmark [13] introduced local mappings between different Poincaré sections to study the grazing-induced bifurcations in an impact oscillator. Luo [14,15] proposed a mapping structure to piece together appropriate or relevant local mappings to form global mappings for a horizontal impact pair system and a ball-bouncing system. Based on Nordmark's work [13], Bernardo et al. [16] derived normal-form mappings to study sliding bifurcations. For an impact system with a drift, Pavlovskaia and Wiercigroch [17] proposed a one-dimensional analytical map to approximate a five-dimensional flow. However, these mapping approaches only consider one non-smoothness (i.e., either impacts or friction) in the system, and the impact system [17] considers the drift in one direction, while the capsule system is subjected to bidirectional drifts, friction and impacts simultaneously. Luo et al. [18] adopted a disturbed map to study the stability of periodic single-impact motions of a unidirectional plastic impact oscillator with a frictional slider. Different from Luo et al. [18], this paper aims to study the dynamics of a bidirectional visco-elastic impact system and to construct global mappings to depict the switching mechanisms between its piecewise smooth subsystems. With a special care of period1 trajectory, Páez Chávez et al. [19] divided the trajectory of the capsule system into a smooth vector field in each disjoint subregion and studied the system by means of path-following techniques. This paper will divide the trajectory of the capsule system in the same way, but consider general periodic motions by studying all possible switching routes in the system.

The rest of this paper is organised as follows. Section 2 studies the modelling of the vibro-impact capsule system. According to the non-smoothness of the system, the 3D map is constructed in Section 3. In Section 4, case studies are provided, and the influence of the amplitude of excitation on the dynamics of the capsule system is investigated. Finally, conclusions are drawn in Section 5.

\section{Modelling of the capsule system}

The two degrees-of-freedom capsule system shown in Fig. 1(b) is considered in this work, where an inner mass $M_{m}$ is connected to a rigid capsule $M_{c}$ via a spring with stiffness $k_{1}$ and a damper with damping coefficient $c$. The inner mass is driven by a harmonic force $P_{d} \cos (\Omega t)$, where $P_{d}, \Omega$ and $t$ represent the amplitude, frequency and time of the excitation, respectively. A secondary spring with stiffness $k_{2}$ is attached to the capsule and the gap between the inner mass and the secondary spring is $G$. $X_{c}$ and $V_{c}$ represent the displacement and velocity of the capsule, whilst $X_{m}$ and $V_{m}$ represent the displacement and velocity of the inner mass, respectively. In this study, Coulomb friction is used to calculate the frictional force between the capsule and the supporting surface,

$$
\begin{cases}F_{f} \in\left[-P_{f}, P_{f}\right], & V_{c}=0, \\ F_{f}=-\operatorname{sign}\left(V_{c}\right) P_{f}, & V_{c} \neq 0,\end{cases}
$$

where $P_{f}=\mu\left(M_{m}+M_{c}\right) g, \mu$ is the friction coefficient between the capsule and the supporting surface, and $g$ is the acceleration due to gravity. As the detailed modelling of the capsule system can be found from [8], we will study the modelling briefly as below.

When the relative displacement between the inner mass and the capsule is smaller than their gap, $X_{m}-X_{c}<G$, the secondary spring is not contacted, and hence the motion of the inner mass is governed by

$M_{m} \ddot{X}_{m}=P_{d} \cos (\Omega t)-k_{1}\left(X_{m}-X_{c}\right)-c\left(\dot{X}_{m}-\dot{X}_{c}\right)$.

The dry friction between the capsule and the supporting surface may lead the capsule to move in stick-slip motion. The capsule is kept still with $\ddot{X}_{c}=0, \dot{X}_{c}=0$ when $\left|k_{1}\left(X_{m}-X_{c}\right)+c\left(\dot{X}_{m}-\dot{X}_{c}\right)\right| \leq$ $P_{f}$. In this situation, the friction force can be determined by the interaction force between the inner mass and the capsule as $F_{f}=$ $-k_{1}\left(X_{m}-X_{c}\right)-c\left(\dot{X}_{m}-\dot{X}_{c}\right)$. Also, the capsule can drift (slip) when $\left|k_{1}\left(X_{m}-X_{c}\right)+c\left(\dot{X}_{m}-\dot{X}_{c}\right)\right|>P_{f}$. So, the motion of the capsule can be expressed as

$M_{c} \ddot{X}_{c}=F_{f}+k_{1}\left(X_{m}-X_{c}\right)+c\left(\dot{X}_{m}-\dot{X}_{c}\right)$,

where the friction force is determined by $F_{f}=-\operatorname{sign}\left(V_{c}\right) P_{f}$.

When the relative displacement is equal to or larger than the gap, $X_{m}-X_{c} \geq G$, the secondary spring is in contact, and the motion of the inner mass can be written as

$M_{m} \ddot{X}_{m}=P_{d} \cos (\Omega t)-k_{1}\left(X_{m}-X_{c}\right)-c\left(\dot{X}_{m}-\dot{X}_{c}\right)-k_{2}\left(X_{m}-X_{c}-G\right)$.

When $\left|k_{1}\left(X_{m}-X_{c}\right)+c\left(\dot{X}_{m}-\dot{X}_{c}\right)\right| \leq P_{f}$, the capsule is kept still with $\ddot{X}_{c}=0, \dot{X}_{c}=0$, and the friction force can be determined by $F_{f}=-k_{1}\left(X_{m}-X_{c}\right)-c\left(\dot{X}_{m}-\dot{X}_{c}\right)-k_{2}\left(X_{m}-X_{c}-G\right)$. When $\left|k_{1}\left(X_{m}-X_{c}\right)+c\left(\dot{X}_{m}-\dot{X}_{c}\right)+k_{2}\left(X_{m}-X_{c}-G\right)\right|>P_{f}$, the capsule may drift forward or backward, governed by

$M_{c} \ddot{X}_{c}=F_{f}+k_{1}\left(X_{m}-X_{c}\right)+c\left(\dot{X}_{m}-\dot{X}_{c}\right)+k_{2}\left(X_{m}-X_{c}-G\right)$,

where the friction force is determined by $F_{f}=-\operatorname{sign}\left(V_{c}\right) P_{f}$. 
Here, for simplicity, we introduce the following non-dimensi onal system parameters,

$\tau=\Omega_{0} t, \omega=\frac{\Omega}{\Omega_{0}}, \alpha=\frac{P_{d}}{P_{f}}, \zeta=\frac{c}{2 M_{m} \Omega_{0}}, \delta=\frac{k_{1}}{P_{f}} G, \beta=\frac{k_{1}}{k_{2}}$,

$\gamma=\frac{M_{c}}{M_{m}}, f_{b}=\frac{\max \left(\left|F_{f}\right|\right)}{P_{f}}$,

to represent the non-dimensional time, excitation frequency and amplitude, damping ratio, gap, stiffness ratio, mass ratio, and friction, respectively, where $\Omega_{0}=\sqrt{\frac{k_{1}}{M_{m}}}$ is the natural frequency of the capsule system. In this study, dry friction is considered, so $f_{b}=1$. The non-dimensional variables of the system can be written as

$x_{c}=\frac{k_{1}}{P_{f}} X_{c}, v_{c}=\frac{\mathrm{d} x_{c}}{\mathrm{~d} \tau}=\frac{k_{1}}{\Omega_{0} P_{f}} X_{c}, \dot{v}_{c}=\frac{\mathrm{d} v_{c}}{\mathrm{~d} \tau}=\frac{k_{1}}{\Omega_{0}^{2} P_{f}} \ddot{X}_{c}$,

$x_{m}=\frac{k_{1}}{P_{f}} X_{m}, v_{m}=\frac{\mathrm{d} x_{m}}{\mathrm{~d} \tau}=\frac{k_{1}}{\Omega_{0} P_{f}} X_{m}$,

$\dot{v}_{m}=\frac{\mathrm{d} v_{m}}{\mathrm{~d} \tau}=\frac{k_{1}}{\Omega_{0}^{2} P_{f}} \ddot{X}_{m}, x_{r}=x_{m}-x_{c}, v_{r}=v_{m}-v_{c}$,

which are the non-dimensional capsule's displacement, velocity and acceleration, inner mass's displacement, velocity and acceleration, the relative displacement and velocity between the inner mass and the capsule, respectively.

Therefore, the equations of motion for the capsule system can be rewritten in a compact form as follows (cf. [8])

$$
\left\{\begin{aligned}
\dot{x}_{m}= & v_{m}, \\
\dot{v}_{m}= & \alpha \cos (\omega \tau)-x_{r}-2 \zeta v_{r}-H_{3} \beta\left(x_{r}-\delta\right), \\
\dot{x}_{c}= & v_{c}, \\
\dot{v}_{c}= & \left(H_{1}\left(1-H_{3}\right)+H_{2} H_{3}\right)\left(-\operatorname{sign}\left(v_{c}\right) f_{b}+x_{r}+2 \zeta v_{r}\right. \\
& \left.+H_{3} \beta\left(x_{r}-\delta\right)\right) / \gamma,
\end{aligned}\right.
$$

with $H_{1}:=H\left(\left|x_{r}+2 \zeta v_{r}\right|-f_{b}\right), H_{2}:=H\left(\left|x_{r}+2 \zeta v_{r}+\beta\left(x_{r}-\delta\right)\right|-\right.$ $\left.f_{b}\right), H_{3}:=H\left(x_{r}-\delta\right)$, where $H(\cdot)$ stands for the Heaviside step function.

We define the state space of the capsule system as $x:=$ $\left(x_{r}, v_{r}, v_{c}\right)$ and introduce four auxiliary functions,

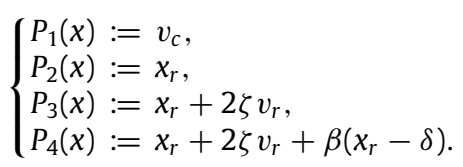

As the capsule system moves in a vibro-impact stick-slip manner, $P_{1}(x)=0$ represents the non-smooth plane induced by dry friction, and $P_{2}(x)=\delta$ represents the non-smooth plane induced by impacts. On the non-smooth plane of $P_{1}(x)=0, P_{3}(x)= \pm f_{b}$ and $P_{4}(x)= \pm f_{b}$ are the boundaries of slip regions.

Next, we divide the trajectories of the piecewise-linear model Eq. (6) by introducing the following six segments.

No contact-forward drift (NC-FD). For $x \in \Omega_{1}:=\left\{x \mid P_{1}(x)>0\right.$, $\left.P_{2}(x) \leq \delta\right\} \cup\left\{x \mid P_{1}(x)=0, P_{2}(x) \leq \delta, P_{3}(x)>f_{b}\right\}$, the equations of motion can be rewritten as

$\dot{x}=F_{1}(x, \tau):=\left(v_{r},-\frac{\gamma+1}{\gamma} P_{3}(x)+\frac{f_{b}}{\gamma}+\alpha \cos (\omega \tau), \frac{P_{3}(x)-f_{b}}{\gamma}\right)$.

No contact-stick (NC-S). For $x \in \Omega_{2}:=\left\{x \mid P_{1}(x)=0, P_{2}(x) \leq\right.$ $\left.\delta,\left|P_{3}(x)\right| \leq f_{b}\right\}$, the capsule system is governed by

$\dot{x}=F_{2}(x, \tau):=\left(v_{r},-P_{3}(x)+\alpha \cos (\omega \tau), 0\right)$.

No contact-backward drift (NC-BD). For $x \in \Omega_{3}:=\left\{x \mid P_{1}(x)<\right.$ $\left.0, P_{2}(x) \leq \delta\right\} \cup\left\{x \mid P_{1}(x)=0, P_{2}(x) \leq \delta, P_{3}(x)<-f_{b}\right\}$, the equations of motion can be written as

$\dot{x}=F_{3}(x, \tau):=\left(v_{r},-\frac{\gamma+1}{\gamma} P_{3}(x)-\frac{f_{b}}{\gamma}+\alpha \cos (\omega \tau), \frac{P_{3}(x)+f_{b}}{\gamma}\right)$.
Contact-forward drift (C-FD). For $x \in \Omega_{4}:=\left\{x \mid P_{1}(x)>0, P_{2}(x)\right.$ $\geq \delta\} \cup\left\{x \mid P_{1}(x)=0, P_{2}(x) \geq \delta, P_{4}(x)>f_{b}\right\}$, the capsule system is governed by

$\dot{x}=F_{4}(x, \tau):=\left(v_{r},-\frac{(\gamma+1)}{\gamma} P_{4}(x)+\frac{f_{b}}{\gamma}+\alpha \cos (\omega \tau), \frac{P_{4}(x)-f_{b}}{\gamma}\right)$.

Contact-stick (C-S). For $x \in \Omega_{5}:=\left\{x \mid P_{1}(x)=0, P_{2}(x) \geq\right.$ $\left.\delta,\left|P_{3}(x)\right| \leq f_{b}\right\}$, the equations of motion of the capsule system can be written as

$\dot{x}=F_{5}(x, \tau):=\left(v_{r},-P_{4}(x)+\alpha \cos (\omega \tau), 0\right)$.

Contact-backward drift (C-BD). For $x \in \Omega_{6}:=\left\{x \mid P_{1}(x)<0\right.$, $\left.P_{2}(x) \geq \delta\right\} \cup\left\{x \mid P_{1}(x)=0, P_{2}(x) \geq \delta, P_{4}(x)<-f_{b}\right\}$, the equations of motion can be written as

$$
\dot{x}=F_{6}(x, \tau):=\left(v_{r},-\frac{(\gamma+1)}{\gamma} P_{4}(x)-\frac{f_{b}}{\gamma}+\alpha \cos (\omega \tau), \frac{P_{4}(x)+f_{b}}{\gamma}\right) .
$$

\section{Switching mechanisms and 3D map}

\subsection{Definitions of boundary surfaces and lines}

According to the non-smooth conditions of the capsule system, the boundary surfaces can be defined as

$$
\left\{\begin{array}{l}
S_{1}:=\left\{x \mid P_{1}(x)=0, P_{2}(x)<\delta, P_{3}(x)>f_{b}\right\}, \\
S_{2}:=\left\{x\left|P_{1}(x)=0, P_{2}(x)<\delta,\right| P_{3}(x) \mid<f_{b}\right\}, \\
S_{3}:=\left\{x \mid P_{1}(x)=0, P_{2}(x)<\delta, P_{3}(x)<-f_{b}\right\}, \\
S_{4}:=\left\{x \mid P_{1}(x)=0, P_{2}(x)>\delta, P_{4}(x)>f_{b}\right\}, \\
S_{5}:=\left\{x\left|P_{1}(x)=0, P_{2}(x)>\delta,\right| P_{4}(x) \mid<f_{b}\right\}, \\
S_{6}:=\left\{x \mid P_{1}(x)=0, P_{2}(x)>\delta, P_{4}(x)<-f_{b}\right\}, \\
S_{7}:=\left\{x \mid P_{1}(x)>0, P_{2}(x)=\delta, v_{r}>0\right\}, \\
S_{8}:=\left\{x \mid P_{1}(x)>0, P_{2}(x)=\delta, v_{r}<0\right\}, \\
S_{9}:=\left\{x \mid P_{1}(x)<0, P_{2}(x)=\delta, v_{r}>0\right\}, \\
S_{10}:=\left\{x \mid P_{1}(x)<0, P_{2}(x)=\delta, v_{r}<0\right\} .
\end{array}\right.
$$

It is worth noting that, the surfaces, $S_{i}$, where $i=1,2, \ldots, 6$, are on the plane $P_{1}(x)=0$ to represent the non-smoothness induced by dry friction. Stick motion of the capsule occurs on the surfaces, $S_{2}$ and $S_{5}$. The surfaces, $S_{i}$, where $i=7,8, \ldots, 10$, are on the plane $P_{2}(x)=\delta$ to denote the non-smoothness induced by visco-elastic impacts. For example, the trajectory of the capsule system enters impact surface through $S_{7}$ or $S_{9}$, and leaves the surface from $S_{8}$ or $S_{10}$.

Among these boundary surfaces, we introduce the following boundary lines to describe different bifurcation events.

$$
\left\{\begin{array}{l}
L_{1}:=\left\{x \mid P_{1}(x)=0, P_{2}(x)<\delta, P_{3}(x)=f_{b}\right\}, \\
L_{2}:=\left\{x \mid P_{1}(x)=0, P_{2}(x) \leq \delta, P_{3}(x)=-f_{b}\right\}, \\
L_{3}:=\left\{x \mid P_{1}(x)=0, P_{2}(x) \geq \delta, P_{4}(x)=f_{b}\right\}, \\
L_{4}:=\left\{x \mid P_{1}(x)=0, P_{2}(x)>\delta, P_{4}(x)=-f_{b}\right\}, \\
L_{5}:=\left\{x \mid P_{1}(x)=0, P_{2}(x)=\delta, v_{r}>-\frac{\delta}{2 \zeta}+\frac{f_{b}}{2 \zeta}\right\}, \\
L_{6}:=\left\{x \mid P_{1}(x)=0, P_{2}(x)=\delta, 0<v_{r}<-\frac{\delta}{2 \zeta}+\frac{f_{b}}{2 \zeta}\right\}, \\
L_{7}:=\left\{x \mid P_{1}(x)=0, P_{2}(x)=\delta,-\frac{\delta}{2 \zeta}-\frac{f_{b}}{2 \zeta}<v_{r}<0\right\}, \\
L_{8}:=\left\{x \mid P_{1}(x)=0, P_{2}(x)=\delta, v_{r}<-\frac{\delta}{2 \zeta}-\frac{f_{b}}{2 \zeta}\right\}, \\
L_{9}:=\left\{x \mid P_{2}(x)=\delta, v_{r}=0\right\} .
\end{array}\right.
$$

According to the vector functions, $F_{n}$, the subspaces, $\Omega_{n}$, where $n=1,2, \ldots, 6$, are divided by the non-smooth boundary surfaces, $S_{i=1,2, \ldots, 10}$, and lines, $L_{j=1,2, \ldots, 9}$, which are visualised in Fig. 2. 

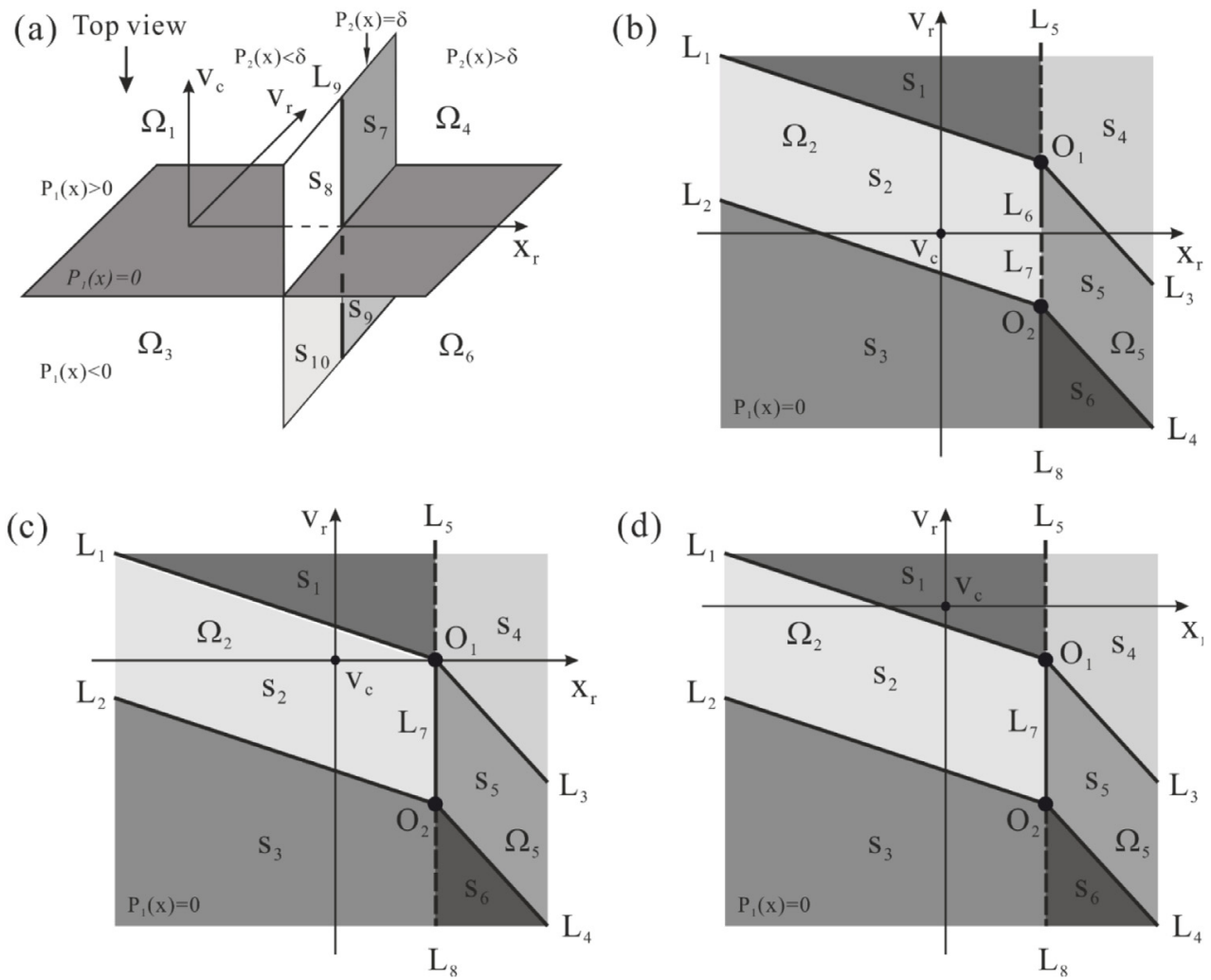

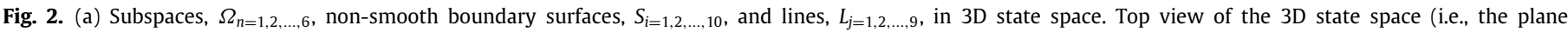

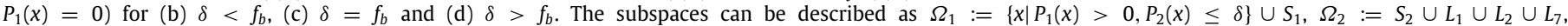
$\Omega_{3}:=\left\{x \mid P_{1}(x)<0, P_{2}(x) \leq \delta\right\} \cup S_{3} \cup L_{8}, \Omega_{4}:=\left\{x \mid P_{1}(x)>0, P_{2}(x) \geq \delta\right\} \cup S_{4} \cup L_{5}, \Omega_{5}:=S_{5} \cup L_{3} \cup L_{4} \cup L_{6}$, and $\Omega_{6}:=\left\{x \mid P_{1}(x)<0, P_{2}(x) \geq \delta\right\} \cup S_{6}$.

So, the subspaces can be expressed as

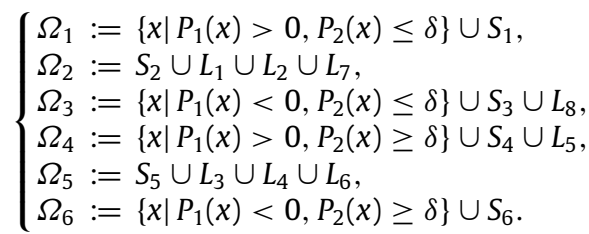

As shown in Fig. 2(b), the intersection of $L_{1}, L_{3}, L_{5}$ and $L_{6}$ is $O_{1}:=\left(\delta, \frac{f_{b}-\delta}{2 \zeta}, 0\right)$, and the intersection of $L_{2}, L_{4}, L_{7}$ and $L_{8}$ is $O_{2}:=\left(\delta,-\frac{f_{b}+\delta}{2 \zeta}, 0\right)$, where we define $O_{1} \in L_{3}$ and $O_{2} \in L_{2}$ for convenience. Since $f_{b} \geq 0$, there are three scenarios for the location of $O_{1}$, i.e., in the top-right quarter, on the axis of $x_{r}^{+}$, and in the bottom-right quarter of the plane $P_{1}(x)=0$ for $\delta<f_{b}, \delta=$ $f_{b}$, and $\delta>f_{b}$, respectively. When $\delta<f_{b}$, if $x(0)=(0,0,0)$, the capsule starts from stick motion and might have drift and impact afterwards. It is noted that $L_{1}, L_{2}, L_{3}$ and $L_{4}$ are the boundary lines separating stick and drifting motions. When a trajectory hits any of these lines, sliding bifurcation may occur. $L_{5}, L_{6}, L_{7}$ and $L_{8}$ are the intersection lines of the non-smooth boundary planes $P_{1}(x)=0$ and $P_{2}(x)=\delta$. When a trajectory hits any of these lines, boundary-intersection crossing bifurcation may occur. Furthermore, we assume that $L_{9}$ is smooth for all vector functions, $F_{n=1,2, \ldots, 6}(x, \tau)$, so $L_{9}$ is not considered as a non-smooth boundary in this study. For $\delta=f_{b}$ and $\delta>f_{b}$, top views of the 3D state space in Fig. 2(a) are presented in Fig. 2(c) and (d). When $\delta=f_{b}, O_{1}:=(\delta, 0,0)$ and if $x(0)=(0,0,0)$, the capsule will start from stick motion and must have forward drift before any impact occurs. For $\delta>f_{b}$, if $x(0)=(0,0,0)$, the capsule will start from forward drift and impact might occur afterwards. It should be noted that, for these two scenarios, $\delta=f_{b}$ and $\delta>f_{b}$, the boundary line $L_{6}$ (for contact-stick motion) doest not exist. For the location of $\mathrm{O}_{2}$, since $-\frac{f_{b}+\delta}{2 \zeta}<0$, it is always in the bottom-right quarter of the plane $P_{1}(x)=0$. In the following subsections, we will use the scenario, $\delta<f_{b}$, as an example to study the switching mechanisms in the 3D map.

\subsection{Switching mechanisms on boundary surfaces}

Let us define the two sides of the surface $S_{i}$ as $S_{i}^{+}$and $S_{i}^{-}$. For $i=1,2, \ldots, 6, S_{i}^{+}$represents the upper half plane of $S_{i}$ at where $P_{1}(x) \rightarrow 0^{+}$, and $S_{i}^{-}$represents its lower half plane for which $P_{1}(x) \rightarrow 0^{-}$. The normals of $S_{i}^{+}$and $S_{i}^{-}$can be written as $\nabla P_{1}^{+}(x)=(0,0,1)$ and $\nabla P_{1}^{-}(x)=(0,0,-1)$, respectively. For $i=7,8,9,10, S_{i}^{+}$represents the right half plane of $S_{i}$ at where $P_{2}(x) \rightarrow \delta^{+}$, and $S_{i}^{-}$represents its left half plane for which $P_{2}(x) \rightarrow \delta^{-}$, so $\nabla P_{2}^{+}(x)=(1,0,0)$ and $\nabla P_{2}^{-}(x)=(-1,0,0)$. Typical examples for the trajectories crossing $S_{1}, S_{5}$ and $S_{7}$ are illustrated in Fig. 3.

As shown in Fig. 3(a), a typical trajectory passes through the boundary surface $S_{1}$ via $x_{1}^{-}$and $x_{1}^{+}$. According to the definition of $S_{1}, P_{3}\left(x_{1}^{-}\right)>f_{b}$ and $P_{3}\left(x_{1}^{+}\right)>f_{b}$. The surface normal for $S_{1}^{-}$ is denoted as $\nabla P_{1}^{-}(x)$, and the trajectory vector is $F_{3}(x, \tau)$. The surface normal for $S_{1}^{+}$is denoted as $\nabla P_{1}^{+}(x)$, and the trajectory vector is $F_{1}(x, \tau)$. So, the approaching direction of this trajectory at $x_{1}^{-}$can be determined by the sign of $\left\langle\nabla P_{1}^{-}(x), F_{3}(x, \tau)\right\rangle$, given as

$$
\left.\left\langle\nabla P_{1}^{-}(x), F_{3}(x, \tau)\right)\right|_{x=x_{1}^{-}}=-\left.\frac{P_{3}(x)+f_{b}}{\gamma}\right|_{x=x_{1}^{-}}<0,
$$

where the negative sign indicates that $S_{1}^{-}$is an attracting surface. The approaching direction of the trajectory at $x_{1}^{+}$can be determined by the sign of $\left\langle\nabla P_{1}^{+}(x), F_{1}(x, \tau)\right)$, given as

$$
\left.\left\langle\nabla P_{1}^{+}(x), F_{1}(x, \tau)\right)\right|_{x=x_{1}^{+}}=\left.\frac{P_{3}(x)-f_{b}}{\gamma}\right|_{x=x_{1}^{+}}>0,
$$



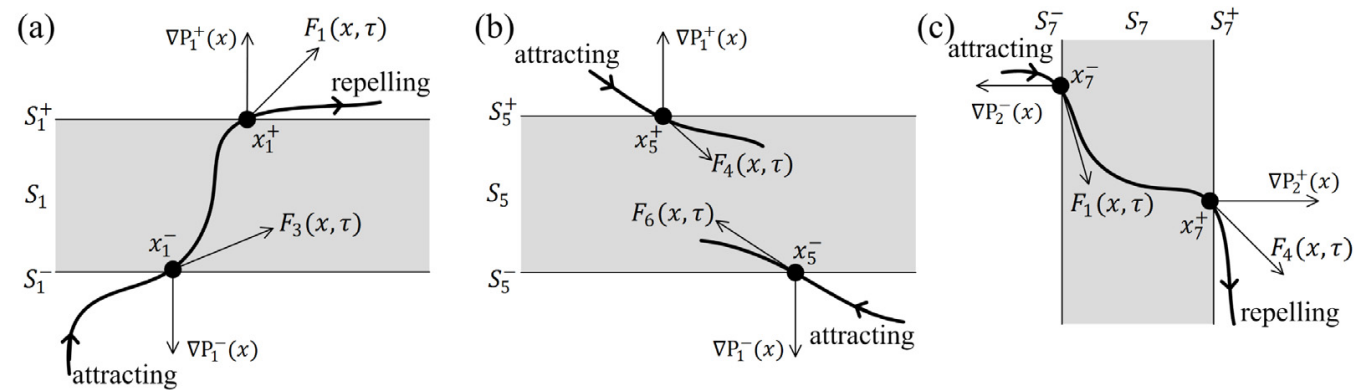

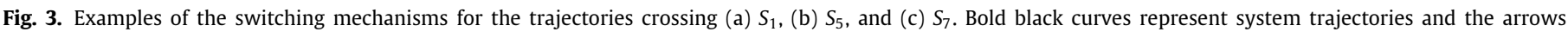

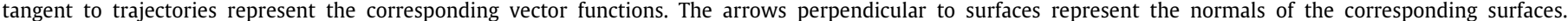

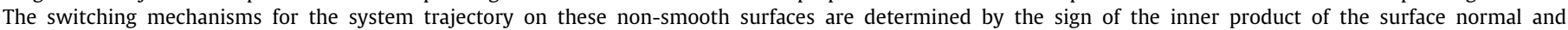

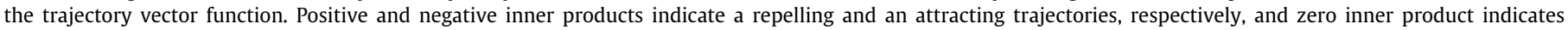
trajectory grazing at boundary surface.

where the positive sign indicates that $S_{1}^{+}$is a repelling surface. Therefore, the switching mechanism on the boundary surface $S_{1}$ is to attract the trajectory from $\Omega_{3}$ and repel it to $\Omega_{1}$. Similar switching mechanisms can be found on the boundary surfaces, $S_{3}, S_{4}$ and $S_{6}$, which are summarised in Table 1.

The second example of the switching mechanism is shown in Fig. 3(b), where a trajectory passes through the boundary surface $S_{5}$ by intersecting $S_{5}^{+}$and $S_{5}^{-}$at $x_{5}^{+}$and $x_{5}^{-}$, respectively. According to the definition of $S_{5}$ in Eq. (13), we can obtain $\left|P_{4}\left(x_{5}^{+}\right)\right|<f_{b}$ and $\left|P_{4}\left(x_{5}^{-}\right)\right|<f_{b}$. Therefore,

$$
\left.\left\langle\nabla P_{1}^{+}(x), F_{4}(x, \tau)\right)\right|_{x=x_{5}^{+}}=\left.\frac{P_{4}(x)-f_{b}}{\gamma}\right|_{x=x_{5}^{+}}<0,
$$

where $\nabla P_{1}^{+}(x)$ is the surface normal of $S_{5}^{+}, F_{4}(x, \tau)$ is the trajectory vector of $x_{5}^{+}$, and

$$
\left.\left\langle\nabla P_{1}^{-}(x), F_{6}(x, \tau)\right)\right|_{x=x_{5}^{-}}=-\left.\frac{P_{4}(x)+f_{b}}{\gamma}\right|_{x=x_{5}^{-}}<0,
$$

where $\nabla P_{1}^{-}(x)$ is the surface normal of $S_{5}^{-}$, and $F_{6}(x, \tau)$ is the trajectory vector of $x_{5}^{-}$. According to Eqs. (18) and (19), both $S_{5}^{+}$ and $S_{5}^{-}$are attracting surfaces, so $S_{5}$ attracts trajectory from both sides of the boundary surface. The switching mechanism on $S_{5}$ is to attract trajectory from $\Omega_{4}$ and $\Omega_{6}$ and repel it to $\Omega_{5}$. The boundary surface $S_{2}$ has similar switching mechanism, and its switching route is given in Table 1.

For the boundary surface $S_{7}$ shown in Fig. 3(c), a trajectory intersects $S_{7}^{-}$and $S_{7}^{+}$at $x_{7}^{-}$and $x_{7}^{+}$, respectively. We can obtain

$$
\left.\left\langle\nabla P_{2}^{-}(x), F_{1}(x, \tau)\right)\right|_{x=x_{7}^{-}}=-v_{r}<0 \text {, }
$$

where $\nabla P_{2}^{-}(x)$ is the surface normal of $S_{7}^{-}, F_{1}(x, \tau)$ is the trajectory vector of $x_{7}^{-}$, and

$$
\left.\left\langle\nabla P_{2}^{+}(x), F_{4}(x, \tau)\right)\right|_{x=x_{7}^{+}}=v_{r}>0,
$$

where $\nabla P_{2}^{+}(x)$ is the surface normal of $S_{7}^{+}$, and $F_{4}(x, \tau)$ is the trajectory vector of $x_{7}^{+}$. According to Eqs. (20) and (21), $S_{7}^{-}$is an attracting surface, and $S_{7}^{+}$is a repelling surface. Therefore, the switching mechanism on $S_{7}$ is to attract trajectory from $\Omega_{1}$ and repel it to $\Omega_{4}$. Similarly, the switching mechanisms on $S_{8}, S_{9}$ and $S_{10}$, which are summarised in Table 1 , can be determined.

\subsection{Switching mechanisms on boundary lines}

When system trajectory hits boundary lines on the plane $P_{1}(x)=0$, four types of sliding bifurcations, including addingsliding, crossing-sliding, grazing-sliding and switching-sliding bifurcations, may occur, which are depicted in Fig. 4. As can be seen from Fig. 4(a), $\nabla P_{3}^{+}(x)=(1,2 \zeta, 0)$ is the normal of $L_{1}$ pointing from $S_{2}$ to $S_{1}, \nabla P_{3}^{-}(x)=(-1,-2 \zeta, 0)$ is the normal of $L_{2}$ pointing from $S_{2}$ to $S_{3}, \nabla P_{4}^{+}(x)=(1+\beta, 2 \zeta, 0)$ is the normal of $L_{3}$ pointing from $S_{5}$ to $S_{4}$, and $\nabla P_{4}^{-}(x)=(-1-\beta,-2 \zeta, 0)$ is the normal of $L_{4}$ pointing from $S_{5}$ to $S_{6}$. The vector of the point $x_{1}$ on $L_{1}$ is given by $F_{2}(x, \tau)$, so

$$
\left.\left\langle\nabla P_{3}^{+}(x), F_{2}(x, \tau)\right)\right|_{x=x_{1}}=v_{r}-2 \zeta f_{b}+2 \zeta \alpha \cos (\omega \tau) \text {. }
$$

For $\alpha \in\left(0, \frac{f_{b}-\delta}{4 \zeta^{2}}-f_{b}\right),\left.\left\langle\nabla P_{3}^{+}(x), F_{2}(x, \tau)\right)\right|_{x=x_{1}}>0$, so $L_{1}$ attracts trajectories from $S_{2}$ and then repels them to $S_{1}$. The section view of $L_{1}$ is shown in Fig. 4(b), where a trajectory hits $L_{1}$ (black dot) from $\Omega_{1}$, and a grazing-sliding bifurcation occurs. If a trajectory hits $L_{1}$ from $\Omega_{3}$, a crossing-sliding bifurcation will occur. Therefore, the switching mechanism on $L_{1}$ is to attract trajectories from $\Omega_{1}, \Omega_{2}$ and $\Omega_{3}$, and repel them to $\Omega_{1}$. Similarly, the switching mechanism on $L_{4}$ is to attract trajectories from $\Omega_{4}, \Omega_{5}$ and $\Omega_{6}$, and repel them to $\Omega_{4}$ for $\alpha \in\left(0, \frac{(1+\beta)\left(f_{b}+\delta\right)}{4 \zeta^{2}}-f_{b}\right)$. In this example, we assume $\alpha \in\left(0, \frac{f_{b}-\delta}{4 \zeta^{2}}-f_{b}\right) \cap\left(0, \frac{(1+\beta)\left(f_{b}+\delta\right)}{4 \zeta^{2}}-f_{b}\right)$, to ensure that the switching directions on $L_{1}$ and $L_{4}$ are unitary. For detailed derivations of these boundaries, one can refer to Appendix A.

For the point $x_{2}$ on the line $L_{2}$, its vector is given by $F_{2}(x, \tau)$, where

$$
\left.\left\langle\nabla P_{3}^{-}(x), F_{2}(x, \tau)\right\rangle\right|_{x=x_{2}}=-v_{r}+2 \zeta f_{b}+2 \zeta \alpha \cos (\omega \tau) .
$$

Based on the amplitude of excitation $\alpha, L_{2}$ can be divided into 3 segments as $L_{2}^{(1)}$ for $v_{r} \in\left[-\frac{f_{b}-\delta}{2 \zeta}, 2 \zeta f_{b}-2 \zeta \alpha\right), L_{2}^{(2)}$ for $v_{r} \in$ $\left[2 \zeta f_{b}-2 \zeta \alpha, 2 \zeta f_{b}+2 \zeta \alpha\right]$, and $L_{2}^{(3)}$ for $v_{r} \in\left(2 \zeta f_{b}+2 \zeta \alpha,+\infty\right)$, which has been detailed in Appendix B. For $L_{2}^{(1)}$, we have $\left\langle\nabla P_{3}^{-}(x)\right.$, $\left.F_{2}(x, \tau)\right)\left.\right|_{x=x_{2}}<0$ and system trajectories are driven out of $S_{2}$. So, the switching mechanism on $L_{2}^{(1)}$ is similar to that on $L_{1}$, attracting trajectories from $\Omega_{1}, \Omega_{2}$ and $\Omega_{3}$, and then repelling them to $\Omega_{3}$. For $L_{2}^{(2)}$, the sign of $\left.\left\langle\nabla P_{3}^{-}(x), F_{2}(x, \tau)\right\rangle\right|_{x=x_{2}}$ depends on time significantly, and consequently, adding-sliding, crossingsliding, grazing-sliding and switching-sliding bifurcations may occur on this boundary line. The switching mechanism on $L_{2}^{(2)}$ is to attract trajectories from $\Omega_{1}, \Omega_{2}$ and $\Omega_{3}$, and then repel them to $\Omega_{2}$ or $\Omega_{3}$. For $L_{2}^{(3)}$, we have $\left.\left\langle\nabla P_{3}^{-}(x), F_{2}(x, \tau)\right)\right|_{x=x_{2}}>0$, and system trajectories will approach to $S_{2}$. As shown in Fig. 4(c), when a trajectory hits $L_{2}^{(3)}$ from $\Omega_{1}$, switching-sliding bifurcation will occur. Therefore, the switching mechanism on $L_{2}^{(3)}$ is to attract trajectories from $\Omega_{1}$ and $\Omega_{3}$, and then repel them to $\Omega_{2}$.

Similarly, for a given amplitude of excitation $\alpha, L_{3}$ can be divided into three segments as $L_{3}^{(1)}$ for $v_{r} \in\left(-\infty, \frac{2 \zeta\left(f_{b}-\alpha\right)}{1+\beta}\right), L_{3}^{(2)}$ for $v_{r} \in\left[\frac{2 \zeta\left(f_{b}-\alpha\right)}{1+\beta}, \frac{2 \zeta\left(f_{b}+\alpha\right)}{1+\beta}\right]$, and $L_{3}^{(3)}$ for $v_{r} \in\left(\frac{2 \zeta\left(f_{b}+\alpha\right)}{1+\beta}, \frac{f_{b}-\delta}{2 \zeta}\right]$, which has been detailed in Appendix B. As a whole, the switching mechanism on $L_{3}$ is to attract trajectories from $\Omega_{4}, \Omega_{5}$ and $\Omega_{6}$, and then 
Table 1

Switching mechanisms on the non-smooth boundary surfaces.

\begin{tabular}{llllll}
\hline Surface & Source space & Target space & Source vector & Target vector & Operation mode \\
\hline$S_{1}$ & $\Omega_{3}$ & $\Omega_{1}$ & $F_{3}(x, \tau)$ & $F_{1}(x, \tau)$ & NC-BD $\rightarrow$ NC-FD \\
\hline \multirow{2}{*}{$S_{2}$} & $\Omega_{1}$ & $\Omega_{2}$ & $F_{1}(x, \tau)$ & $F_{2}(x, \tau)$ & NC-FD $\rightarrow$ NC-S \\
& $\Omega_{3}$ & $\Omega_{2}$ & $F_{3}(x, \tau)$ & $F_{2}(x, \tau)$ & NC-BD $\rightarrow$ NC-S \\
\hline$S_{3}$ & $\Omega_{1}$ & $\Omega_{3}$ & $F_{1}(x, \tau)$ & $F_{3}(x, \tau)$ & NC-FD $\rightarrow$ NC-BD \\
\hline$S_{4}$ & $\Omega_{6}$ & $\Omega_{4}$ & $F_{6}(x, \tau)$ & $F_{4}(x, \tau)$ & C-BD $\rightarrow$ C-FD \\
\hline$S_{5}$ & $\Omega_{4}$ & $\Omega_{5}$ & $F_{4}(x, \tau)$ & $F_{5}(x, \tau)$ & C-FD $\rightarrow$ C-S \\
\hline$S_{6}$ & $\Omega_{6}$ & $\Omega_{5}$ & $F_{6}(x, \tau)$ & $F_{5}(x, \tau)$ & C-BD $\rightarrow$ C-S \\
\hline$S_{7}$ & $\Omega_{4}$ & $\Omega_{6}$ & $F_{4}(x, \tau)$ & $F_{6}(x, \tau)$ & C-FD $\rightarrow$ C-BD \\
\hline$S_{8}$ & $\Omega_{4}$ & $\Omega_{4}$ & $F_{1}(x, \tau)$ & $F_{4}(x, \tau)$ & NC-FD $\rightarrow$ C-FD \\
\hline$S_{9}$ & $\Omega_{3}$ & $\Omega_{1}$ & $F_{4}(x, \tau)$ & $F_{1}(x, \tau)$ & C-FD $\rightarrow$ NC-FD \\
\hline$S_{10}$ & $\Omega_{6}$ & $\Omega_{6}$ & $F_{3}(x, \tau)$ & $F_{6}(x, \tau)$ & NC-BD $\rightarrow$ C-BD \\
\hline & $\Omega_{3}$ & $F_{6}(x, \tau)$ & $F_{3}(x, \tau)$ & C-BD $\rightarrow$ NC-BD \\
\hline
\end{tabular}

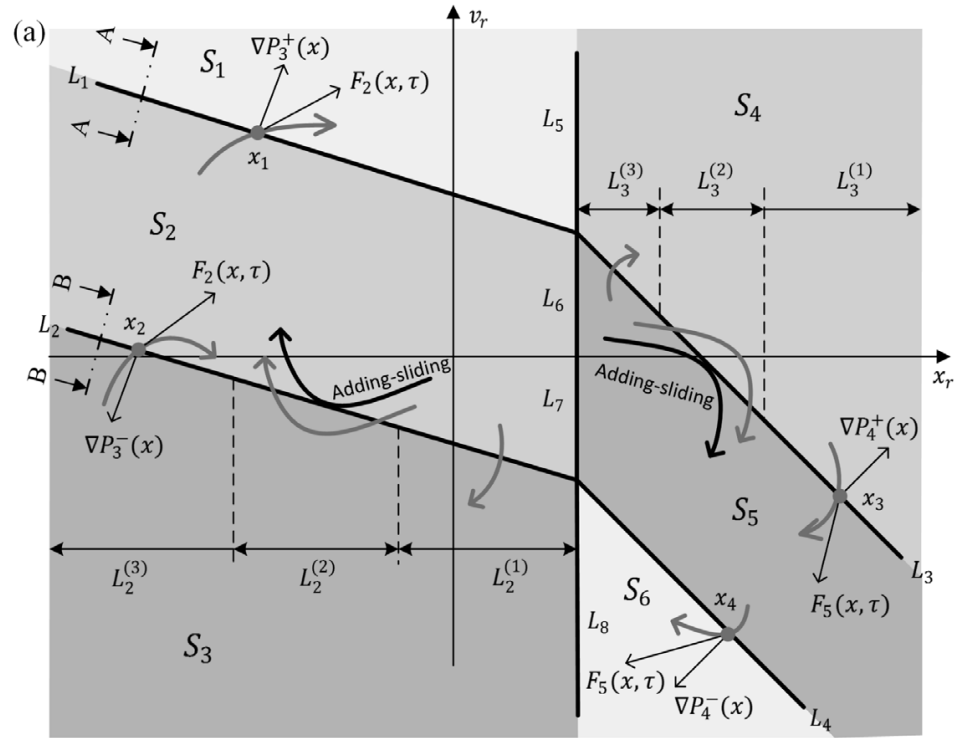

(b) Section View A

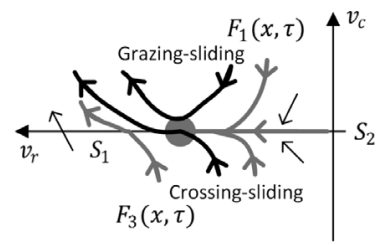

(c) Section View B

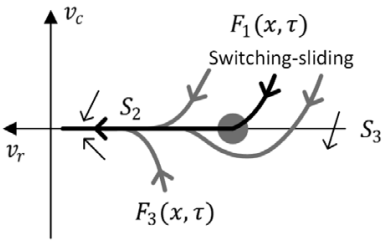

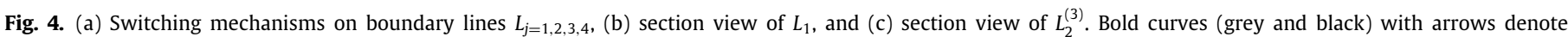

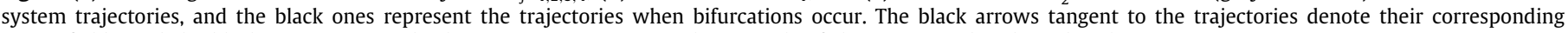
vector fields, and the black arrows perpendicular to $L_{j=1,2,3,4}$ represent the normals of the corresponding boundary lines.

repel them to $\Omega_{4}$ or $\Omega_{5}$. So, switching-sliding bifurcations can occur on $L_{3}^{(1)}$, all the four types of sliding bifurcations can happen on $L_{3}^{(2)}$, and crossing-sliding and grazing-sliding bifurcations may exist on $L_{3}^{(3)}$.

The switching mechanisms and possible bifurcations on the boundary lines $L_{j=5,6,7,8}$ are shown in Fig. 5, where section view of each boundary line is presented in Fig. 5(b)-(e). As can be seen from Fig. 5(b), the boundary line $L_{5}$ degenerates to a point at where the boundary-intersection crossing bifurcation will occur when system trajectory hits $L_{5}$. The switching mechanism for $L_{5}$ is to attract trajectories from $\Omega_{3}$ and then repel them to $\Omega_{4}$. Similarly, as shown in Fig. 5(e), the passage of $L_{8}$ indicates the occurrence of a boundary-intersection crossing bifurcation from $\Omega_{4}$ to $\Omega_{3}$. As can be seen from Fig. 5(c), when trajectories hit $L_{6}$ from $\Omega_{1}$ and $\Omega_{3}$, boundary-intersection crossing bifurcations will occur, and both trajectories enter $S_{5}$. Therefore, the switching mechanism on $L_{6}$ is to attract trajectories from $\Omega_{1}, \Omega_{2}$ and $\Omega_{3}$, and then repel them to $\Omega_{5}$. Opposite to $L_{6}$, as shown in Fig. $5(\mathrm{~d})$, the switching mechanism on $L_{7}$ is to attract trajectories from $\Omega_{4}$, $\Omega_{5}$ and $\Omega_{6}$, and then repel them to $\Omega_{2}$.

Finally, the switching mechanisms on $L_{j=1,2, \ldots, 8}$ are summarised in Table 2. It is worth noting that the boundary line $L_{9}$ satisfies the condition of grazing bifurcations. However, we have defined the vector functions $F_{n=1,2, \ldots, 6}$ are smooth on $L_{9}$. It is therefore that $L_{9}$ will not be considered as a non-smooth boundary in this study.

\section{4. $3 D$ map}

Based on the switching mechanisms on the boundary surfaces and lines, we can define the following switching planes for the 3D map.

$$
\left\{\begin{aligned}
\Sigma_{1} & :=S_{1}, \\
\Sigma_{2} & :=S_{2} \cup L_{1} \cup L_{2} \cup L_{7}, \\
\Sigma_{3} & :=S_{3} \cup L_{8}, \\
\Sigma_{4} & :=S_{4} \cup L_{5}, \\
\Sigma_{5} & :=S_{5} \cup L_{3} \cup L_{4} \cup L_{6}, \\
\Sigma_{6} & :=S_{6}, \\
\Sigma_{7} & :=S_{7}, \\
\Sigma_{8} & :=S_{8}, \\
\Sigma_{9} & :=S_{9}, \\
\Sigma_{10} & :=S_{10} .
\end{aligned}\right.
$$

According to these switching planes, local mapping, $P: \Sigma_{k} \rightarrow \Sigma_{i}$ given by $F_{n}(x, \tau)$, can be written as $P_{\Sigma_{i}, F_{n}, \Sigma_{k}}$, where $\Sigma_{i=1,2, \ldots, 10}$, $F_{n=1,2, \ldots, 6}$ and $\Sigma_{k=1,2, \ldots, 10}$ represent the target switching plane, the vector function, and the source switching plane, respectively. In 


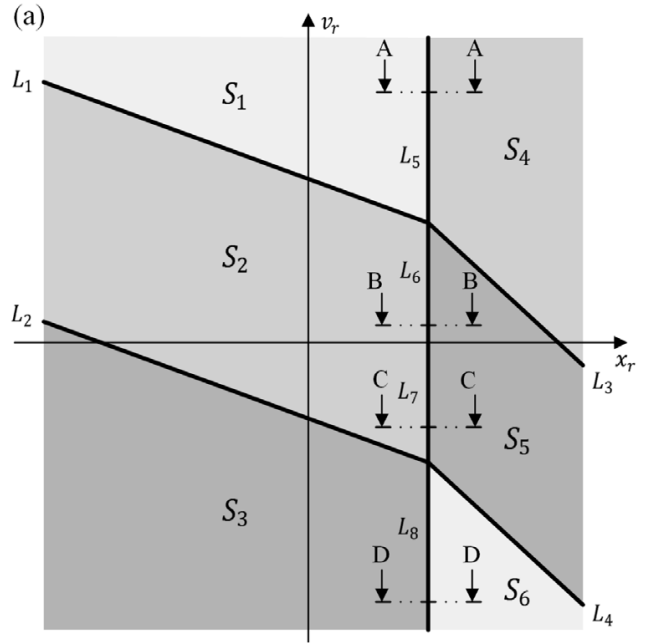

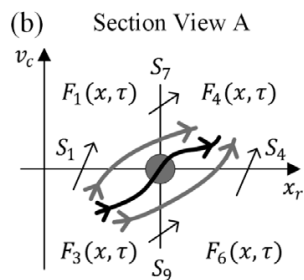

(d) Section View C

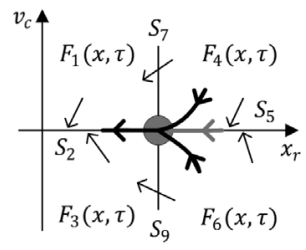

(c) Section View B

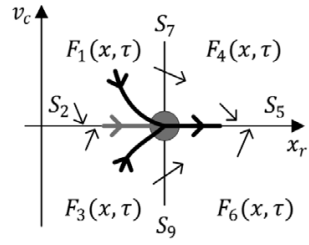

(e) Section View D

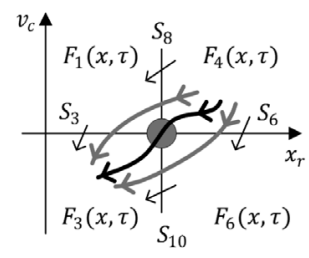

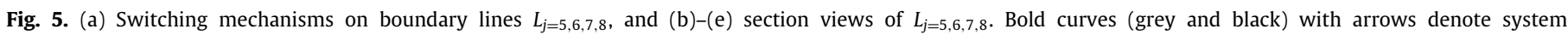
trajectories, and the black ones represent the trajectories when bifurcations occur.

Table 2

Switching mechanisms on the non-smooth boundary lines.

\begin{tabular}{|c|c|c|c|c|c|}
\hline Lines & Source spaces & Target spaces & Source vectors & Target vectors & Operation mode \\
\hline \multirow{3}{*}{$L_{1}$} & $\Omega_{1}$ & $\Omega_{1}$ & $F_{1}(x, \tau)$ & $F_{1}(x, \tau)$ & $\mathrm{NC}-\mathrm{FD} \rightarrow \mathrm{NC}-\mathrm{FD}$ \\
\hline & $\Omega_{2}$ & $\Omega_{1}$ & $F_{2}(x, \tau)$ & $F_{1}(x, \tau)$ & $\mathrm{NC}-\mathrm{S} \rightarrow \mathrm{NC}-\mathrm{FD}$ \\
\hline & $\Omega_{3}$ & $\Omega_{1}$ & $F_{3}(x, \tau)$ & $F_{1}(x, \tau)$ & NC-BD $\rightarrow$ NC-FD \\
\hline \multirow{6}{*}{$L_{2}$} & $\Omega_{1}$ & $\Omega_{2}$ & $F_{1}(x, \tau)$ & $F_{2}(x, \tau)$ & $\mathrm{NC}-\mathrm{FD} \rightarrow \mathrm{NC}-\mathrm{S}$ \\
\hline & $\Omega_{2}$ & $\Omega_{2}$ & $F_{2}(x, \tau)$ & $F_{2}(x, \tau)$ & $\mathrm{NC}-\mathrm{S} \rightarrow \mathrm{NC}-\mathrm{S}$ \\
\hline & $\Omega_{3}$ & $\Omega_{2}$ & $F_{3}(x, \tau)$ & $F_{2}(x, \tau)$ & $\mathrm{NC}-\mathrm{BD} \rightarrow \mathrm{NC}-\mathrm{S}$ \\
\hline & $\Omega_{1}$ & $\Omega_{3}$ & $F_{1}(x, \tau)$ & $F_{3}(x, \tau)$ & $\mathrm{NC}-\mathrm{FD} \rightarrow \mathrm{NC}-\mathrm{BD}$ \\
\hline & $\Omega_{2}$ & $\Omega_{3}$ & $F_{2}(x, \tau)$ & $F_{3}(x, \tau)$ & $\mathrm{NC}-\mathrm{S} \rightarrow \mathrm{NC}-\mathrm{BD}$ \\
\hline & $\Omega_{3}$ & $\Omega_{3}$ & $F_{3}(x, \tau)$ & $F_{3}(x, \tau)$ & $\mathrm{NC}-\mathrm{BD} \rightarrow \mathrm{NC}-\mathrm{BD}$ \\
\hline \multirow{6}{*}{$L_{3}$} & $\Omega_{4}$ & $\Omega_{4}$ & $F_{4}(x, \tau)$ & $F_{4}(x, \tau)$ & C-FD $\rightarrow$ C-FD \\
\hline & $\Omega_{5}$ & $\Omega_{4}$ & $F_{5}(x, \tau)$ & $F_{4}(x, \tau)$ & $\mathrm{C}-\mathrm{S} \rightarrow \mathrm{C}-\mathrm{FD}$ \\
\hline & $\Omega_{6}$ & $\Omega_{4}$ & $F_{6}(x, \tau)$ & $F_{4}(x, \tau)$ & $\mathrm{C}-\mathrm{BD} \rightarrow \mathrm{C}-\mathrm{FD}$ \\
\hline & $\Omega_{4}$ & $\Omega_{5}$ & $F_{4}(x, \tau)$ & $F_{5}(x, \tau)$ & $\mathrm{C}-\mathrm{FD} \rightarrow \mathrm{C}-\mathrm{S}$ \\
\hline & $\Omega_{5}$ & $\Omega_{5}$ & $F_{5}(x, \tau)$ & $F_{5}(x, \tau)$ & $\mathrm{C}-\mathrm{S} \rightarrow \mathrm{C}-\mathrm{S}$ \\
\hline & $\Omega_{6}$ & $\Omega_{5}$ & $F_{6}(x, \tau)$ & $F_{5}(x, \tau)$ & $\mathrm{C}-\mathrm{BD} \rightarrow \mathrm{C}-\mathrm{S}$ \\
\hline \multirow{3}{*}{$L_{4}$} & $\Omega_{4}$ & $\Omega_{4}$ & $F_{4}(x, \tau)$ & $F_{4}(x, \tau)$ & C-FD $\rightarrow$ C-FD \\
\hline & $\Omega_{5}$ & $\Omega_{4}$ & $F_{5}(x, \tau)$ & $F_{4}(x, \tau)$ & $\mathrm{C}-\mathrm{S} \rightarrow \mathrm{C}-\mathrm{FD}$ \\
\hline & $\Omega_{6}$ & $\Omega_{4}$ & $F_{6}(x, \tau)$ & $F_{4}(x, \tau)$ & $\mathrm{C}-\mathrm{BD} \rightarrow \mathrm{C}-\mathrm{FD}$ \\
\hline$L_{5}$ & $\Omega_{3}$ & $\Omega_{4}$ & $F_{3}(x, \tau)$ & $F_{4}(x, \tau)$ & $\mathrm{NC}-\mathrm{BD} \rightarrow \mathrm{C}-\mathrm{FD}$ \\
\hline \multirow{3}{*}{$L_{6}$} & $\Omega_{1}$ & $\Omega_{5}$ & $F_{1}(x, \tau)$ & $F_{5}(x, \tau)$ & $\mathrm{NC}-\mathrm{FD} \rightarrow \mathrm{C}-\mathrm{S}$ \\
\hline & $\Omega_{2}$ & $\Omega_{5}$ & $F_{2}(x, \tau)$ & $F_{5}(x, \tau)$ & $\mathrm{NC}-\mathrm{S} \rightarrow \mathrm{C}-\mathrm{S}$ \\
\hline & $\Omega_{3}$ & $\Omega_{5}$ & $F_{3}(x, \tau)$ & $F_{5}(x, \tau)$ & $\mathrm{NC}-\mathrm{BD} \rightarrow \mathrm{C}-\mathrm{S}$ \\
\hline \multirow{3}{*}{$L_{7}$} & $\Omega_{4}$ & $\Omega_{2}$ & $F_{4}(x, \tau)$ & $F_{2}(x, \tau)$ & $\mathrm{C}-\mathrm{FD} \rightarrow \mathrm{NC}-\mathrm{S}$ \\
\hline & $\Omega_{5}$ & $\Omega_{2}$ & $F_{5}(x, \tau)$ & $F_{2}(x, \tau)$ & $\mathrm{C}-\mathrm{S} \rightarrow \mathrm{NC}-\mathrm{S}$ \\
\hline & $\Omega_{6}$ & $\Omega_{2}$ & $F_{6}(x, \tau)$ & $F_{2}(x, \tau)$ & $\mathrm{C}-\mathrm{BD} \rightarrow \mathrm{NC}-\mathrm{S}$ \\
\hline$L_{8}$ & $\Omega_{4}$ & $\Omega_{3}$ & $F_{4}(x, \tau)$ & $F_{3}(x, \tau)$ & $\mathrm{C}-\mathrm{FD} \rightarrow \mathrm{NC}-\mathrm{BD}$ \\
\hline
\end{tabular}

summary, all possible 3D local mappings for the capsule system are given in Table 3.

\section{Numerical studies}

Numerical studies by varying the amplitude of excitation are given in this section to show how the proposed 3D map can be used to describe and explain the dynamics of the capsule system with a focus on its switching mechanisms on the non-smooth boundaries. The overall influence of $\alpha$ on the dynamics of the capsule system is presented in Fig. 6, calculated for $\omega=0.8$, $\delta=0.02, \zeta=0.05, \beta=1.5$ and $\gamma=3.3$. As $\alpha$ increases from 0 to 2 , grazing, adding-sliding, boundary-intersection crossing, crossing-sliding, switching-sliding and grazing-sliding bifurcations can be observed.

Based on various bifurcations encountered when the amplitude of excitation increases, dynamics of the capsule system is considered from the following regimes. For each typical trajectory, the displacements of the inner mass and the capsule are shown in Fig. 7, together with its phase portrait, to explain how the orbit switches from one non-smooth boundary to another. The dynamics of the capsule system for each regime is summarised as follows.

- $\alpha \in(0,0.0074]$ : As shown in Fig. 7(a), the global mapping of the capsule system can be represented by an identity mapping, $P=I d_{\Sigma_{2}, F_{2}, \Sigma_{2}}$, which is dominated by $F_{2}(x, \tau)$, and the capsule is kept stationary without any impact. For $\alpha=0.0074$, a grazing bifurcation is encountered, and the system trajectory passes $L_{9}$ tangentially. Further increase of $\alpha$ will lead to the occurrence of impacts.

- $\alpha \in(0.0074,0.3687]$ : As shown in Fig. 7(b), the global mapping can be written as $P=P_{\Sigma_{5}, F_{2}, \Sigma_{2}} \circ P_{\Sigma_{2}, F_{5}, \Sigma_{5}}$. The capsule is kept stationary with one impact per period of excitation. 
Table 3

All 3D local mappings for the capsule system.

\begin{tabular}{|c|c|c|c|c|c|}
\hline Mapping & Operation & Vector & Mapping & Operation & Vector \\
\hline$P_{\Sigma_{2}, F_{1}, \Sigma_{1}}$ & $\Sigma_{2} \stackrel{F_{1}}{\leftarrow} \Sigma_{1}$ & NC-FD & $P_{\Sigma_{2}, F_{1}, \Sigma_{8}}$ & $\Sigma_{2} \stackrel{F_{1}}{\leftarrow} \Sigma_{8}$ & NC-FD \\
\hline$P_{\Sigma_{3}, F_{1}, \Sigma_{1}}$ & $\Sigma_{3} \stackrel{F_{1}}{\longleftarrow} \Sigma_{1}$ & NC-FD & $P_{\Sigma_{3}, F_{1}, \Sigma_{8}}$ & $\Sigma_{3} \stackrel{F_{1}}{\leftarrow} \Sigma_{8}$ & NC-FD \\
\hline$P_{\Sigma_{5}, F_{1}, \Sigma_{1}}$ & $\Sigma_{5} \stackrel{F_{1}}{\longleftarrow} \Sigma_{1}$ & NC-FD & $P_{\Sigma_{5}, F_{1}, \Sigma_{8}}$ & $\Sigma_{5} \stackrel{F_{1}}{\leftarrow} \Sigma_{8}$ & NC-FD \\
\hline$P_{\Sigma_{7}, F_{1}, \Sigma_{1}}$ & $\Sigma_{7} \stackrel{F_{1}}{\longleftarrow} \Sigma_{1}$ & NC-FD & $P_{\Sigma_{7}, F_{1}, \Sigma_{8}}$ & $\Sigma_{7} \stackrel{F_{1}}{\leftarrow} \Sigma_{8}$ & NC-FD \\
\hline$P_{\Sigma_{1}, F_{2}, \Sigma_{2}}$ & $\Sigma_{1} \stackrel{F_{2}}{\longleftarrow} \Sigma_{2}$ & NC-S & $P_{\Sigma_{2}, F_{5}, \Sigma_{5}}$ & $\Sigma_{2} \stackrel{F_{5}}{\leftarrow} \Sigma_{5}$ & C-S \\
\hline$P_{\Sigma_{3}, F_{2}, \Sigma_{2}}$ & $\Sigma_{3} \stackrel{F_{2}}{\longleftarrow} \Sigma_{2}$ & NC-S & $P_{\Sigma_{4}, F_{5}, \Sigma_{5}}$ & $\Sigma_{4} \stackrel{F_{5}}{\leftarrow} \Sigma_{5}$ & C-S \\
\hline$P_{\Sigma_{5}, F_{2}, \Sigma_{2}}$ & $\Sigma_{5} \stackrel{F_{2}}{\longleftarrow} \Sigma_{2}$ & NC-S & $P_{\Sigma_{6}, F_{5}, \Sigma_{5}}$ & $\Sigma_{6} \stackrel{F_{5}}{\leftarrow} \Sigma_{5}$ & C-S \\
\hline$P_{\Sigma_{1}, F_{3}, \Sigma_{3}}$ & $\Sigma_{1} \stackrel{F_{3}}{\leftarrow} \Sigma_{3}$ & NC-BD & $P_{\Sigma_{1}, F_{3}, \Sigma_{10}}$ & $\Sigma_{1} \stackrel{F_{3}}{\leftarrow} \Sigma_{10}$ & NC-BD \\
\hline$P_{\Sigma_{2}, F_{3}, \Sigma_{3}}$ & $\Sigma_{2} \stackrel{F_{3}}{\longleftarrow} \Sigma_{3}$ & NC-BD & $P_{\Sigma_{2}, F_{3}, \Sigma_{10}}$ & $\Sigma_{2} \stackrel{F_{3}}{\longleftarrow} \Sigma_{10}$ & NC-BD \\
\hline$P_{\Sigma_{4}, F_{3}, \Sigma_{3}}$ & $\Sigma_{4} \stackrel{F_{3}}{\leftarrow} \Sigma_{3}$ & NC-BD & $P_{\Sigma_{4}, F_{3}, \Sigma_{10}}$ & $\Sigma_{4} \stackrel{F_{3}}{\leftarrow} \Sigma_{10}$ & NC-BD \\
\hline$P_{\Sigma_{5}, F_{3}, \Sigma_{3}}$ & $\Sigma_{5} \stackrel{F_{3}}{\longleftarrow} \Sigma_{3}$ & NC-BD & $P_{\Sigma_{5}, F_{3}, \Sigma_{10}}$ & $\Sigma_{5} \stackrel{F_{3}}{\longleftarrow} \Sigma_{10}$ & NC-BD \\
\hline$P_{\Sigma_{9}, F_{3}, \Sigma_{3}}$ & $\Sigma_{9} \stackrel{F_{3}}{\longleftarrow} \Sigma_{3}$ & NC-BD & $P_{\Sigma_{9}, F_{3}, \Sigma_{10}}$ & $\Sigma_{9} \stackrel{F_{3}}{\leftarrow} \Sigma_{10}$ & NC-BD \\
\hline$P_{\Sigma_{2}, F_{4}, \Sigma_{4}}$ & $\Sigma_{2} \stackrel{F_{4}}{\leftarrow} \Sigma_{4}$ & C-FD & $P_{\Sigma_{2}, F_{4}, \Sigma_{7}}$ & $\Sigma_{2} \stackrel{F_{4}}{\leftarrow} \Sigma_{7}$ & C-FD \\
\hline$P_{\Sigma_{3}, F_{4}, \Sigma_{4}}$ & $\Sigma_{3} \stackrel{F_{4}}{\leftarrow} \Sigma_{4}$ & C-FD & $P_{\Sigma_{3}, F_{4}, \Sigma_{7}}$ & $\Sigma_{3} \stackrel{F_{4}}{\longleftarrow} \Sigma_{7}$ & C-FD \\
\hline$P_{\Sigma_{5}, F_{4}, \Sigma_{4}}$ & $\Sigma_{5} \stackrel{F_{4}}{\longleftarrow} \Sigma_{4}$ & C-FD & $P_{\Sigma_{5}, F_{4}, \Sigma_{7}}$ & $\Sigma_{5} \stackrel{F_{4}}{\longleftarrow} \Sigma_{7}$ & C-FD \\
\hline$P_{\Sigma_{6}, F_{4}, \Sigma_{4}}$ & $\Sigma_{6} \stackrel{F_{4}}{\longleftarrow} \Sigma_{4}$ & C-FD & $P_{\Sigma_{6}, F_{4}, \Sigma_{7}}$ & $\Sigma_{6} \stackrel{F_{4}}{\longleftarrow} \Sigma_{7}$ & C-FD \\
\hline$P_{\Sigma_{8}, F_{4}, \Sigma_{4}}$ & $\Sigma_{8} \stackrel{F_{4}}{\leftarrow} \Sigma_{4}$ & C-FD & $P_{\Sigma_{8}, F_{4}, \Sigma_{7}}$ & $\Sigma_{8} \stackrel{F_{4}}{\longleftarrow} \Sigma_{7}$ & C-FD \\
\hline$P_{\Sigma_{2}, F_{6}, \Sigma_{6}}$ & $\Sigma_{2} \stackrel{F_{6}}{\leftarrow} \Sigma_{6}$ & C-BD & $P_{\Sigma_{2}, F_{6}, \Sigma_{9}}$ & $\Sigma_{2} \stackrel{F_{6}}{\leftarrow} \Sigma_{9}$ & C-BD \\
\hline$P_{\Sigma_{4}, F_{6}, \Sigma_{6}}$ & $\Sigma_{4} \stackrel{F_{6}}{\leftarrow} \Sigma_{6}$ & C-BD & $P_{\Sigma_{4}, F_{6}, \Sigma_{9}}$ & $\Sigma_{4} \stackrel{F_{6}}{\leftarrow} \Sigma_{9}$ & C-BD \\
\hline$P_{\Sigma_{5}, F_{6}, \Sigma_{6}}$ & $\Sigma_{5} \stackrel{F_{6}}{\leftarrow} \Sigma_{6}$ & C-BD & $P_{\Sigma_{5}, F_{6}, \Sigma_{9}}$ & $\Sigma_{5} \stackrel{F_{6}}{\leftarrow} \Sigma_{9}$ & C-BD \\
\hline$P_{\Sigma_{10}, F_{6}, \Sigma_{6}}$ & $\Sigma_{10} \stackrel{F_{6}}{\leftarrow} \Sigma_{6}$ & C-BD & $P_{\Sigma_{10}, F_{6}, \Sigma_{9}}$ & $\Sigma_{10} \stackrel{F_{6}}{\leftarrow} \Sigma_{9}$ & C-BD \\
\hline
\end{tabular}

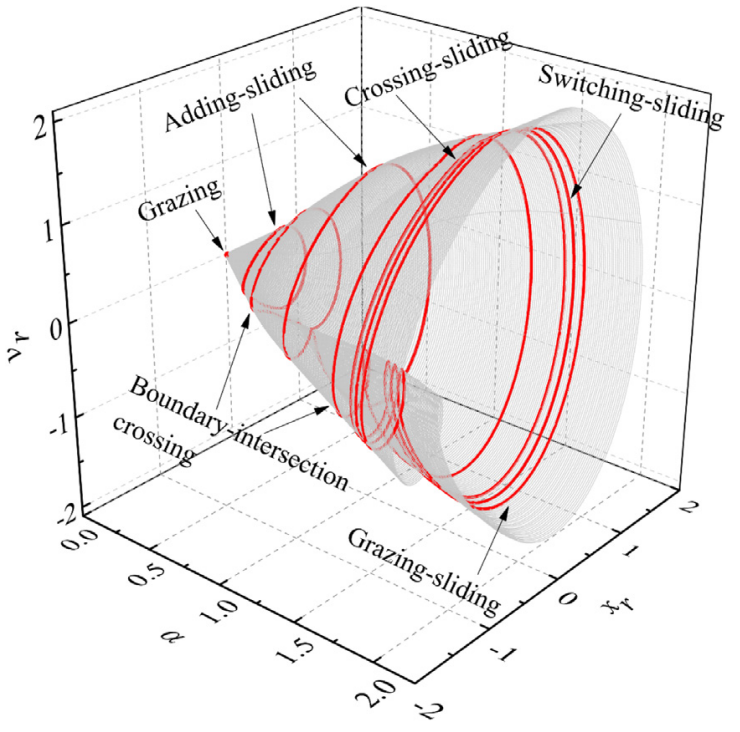

Fig. 6. Evolution of phase trajectories of the capsule system in the state space $\left(x_{r}, v_{r}\right)$ under variation of the amplitude of excitation $\alpha \in[0,2]$. As $\alpha$ increases, grazing, adding-sliding, boundary-intersection crossing, crossing-sliding, switching-sliding and grazing-sliding bifurcations are observed. Simulation conditions are $\omega=0.8, \delta=0.02, \zeta=0.05, \beta=1.5$, and $\gamma=3.3$ by using initial state $\left(x_{r}, v_{r}, v_{c}\right)=(0,0,0)$. (For interpretation of the references to colour in this figure legend, the reader is referred to the web version of this article.)

When $\alpha=0.3687$, as shown in Fig. 6(c), an adding-sliding bifurcation occurs, and the trajectory (the black segment) passes $L_{3}^{(2)}$ tangentially. As $\alpha$ further increases, the capsule will move forward.
- $\alpha \in(0.3687,0.5245)$ : As shown in Fig. 7(d), the global mapping of the capsule system can be represented by $P=$ $P_{\Sigma_{5}, F_{2}, \Sigma_{2}} \circ P_{\Sigma_{2}, F_{5}, \Sigma_{5}} \circ P_{\Sigma_{5}, F_{4}, \Sigma_{4}} \circ P_{\Sigma_{4}, F_{5}, \Sigma_{5}}$, where the local mapping $P_{\Sigma_{5}, F_{4}, \Sigma_{4}}$ indicates a forward drift of the capsule.

- $\alpha=0.5245$ : A boundary-intersection crossing bifurcation occurs as shown in Fig. 7(e), when the trajectory (the green segment) hits and passes through $L_{7}$ from $\Sigma_{4}$ to $\Sigma_{2}$ directly. Therefore, the global mapping can be represented as $P=$ $P_{\Sigma_{5}, F_{2}, \Sigma_{2}} \circ P_{\Sigma_{2}, F_{4}, \Sigma_{4}} \circ P_{\Sigma_{4}, F_{5}, \Sigma_{5}}$. Further increase of $\alpha$ will make the trajectory leave the impact boundary through $S_{8}$. The 3D trajectories for the boundary-intersection crossing bifurcation around $\alpha=0.5245$ are shown in Fig. 8(a) and (b).

- $\alpha \in(0.5245,0.939]$ : As shown in Fig. 7(f), the global mapping for this regime can be written as $P=P_{\Sigma_{5}, F_{2}, \Sigma_{2}} \circ$ $P_{\Sigma_{2}, F_{1}, \Sigma_{8}} \circ P_{\Sigma_{8}, F_{4}, \Sigma_{4}} \circ P_{\Sigma_{4}, F_{5}, \Sigma_{5}}$. For the segments of $F_{4}(x, \tau)$ and $F_{1}(x, \tau)$, the capsule moves forward without and with impact, respectively. For $\alpha=0.939$ as shown in Fig. 7(g), an adding-sliding bifurcation is encountered, and the trajectory (the red segment) passes $L_{2}$ tangentially. As $\alpha$ increases further, backward motion of the capsule can be observed. A detailed adding-sliding bifurcation around $\alpha=0.939$ can be found in Fig. 9(a).

- $\alpha \in(0.939,1.443)$ : The global mapping for this regime can be represented as $P=P_{\Sigma_{5}, F_{2}, \Sigma_{2}} \circ P_{\Sigma_{2}, F_{3}, \Sigma_{3}} \circ P_{\Sigma_{3}, F_{2}, \Sigma_{2}} \circ$ $P_{\Sigma_{2}, F_{1}, \Sigma_{8}} \circ P_{\Sigma_{8}, F_{4}, \Sigma_{4}} \circ P_{\Sigma_{4}, F_{5}, \Sigma_{5}}$. As can be seen from the trajectory shown in Fig. 7(h), the magenta segment for backward drift dominated by $F_{3}(x, \tau)$ is inserted between the red segments dominated by $F_{2}(x, \tau)$.

- $\alpha=$ 1.443: A boundary-intersection crossing bifurcation occurs at $\alpha=1.443$, when the trajectory (the magenta segment) hits $L_{6}$ as shown in Fig. $7(\mathrm{i})$, at where $P_{\Sigma_{5}, F_{3}, \Sigma_{3}}$ maps the trajectory from $\Sigma_{3}$ into $\Sigma_{5}$ directly. A detailed 3D trajectory for this bifurcation around $\alpha=1.443$ can be found from Fig. 8(c) and (d). The global mapping can be given as 

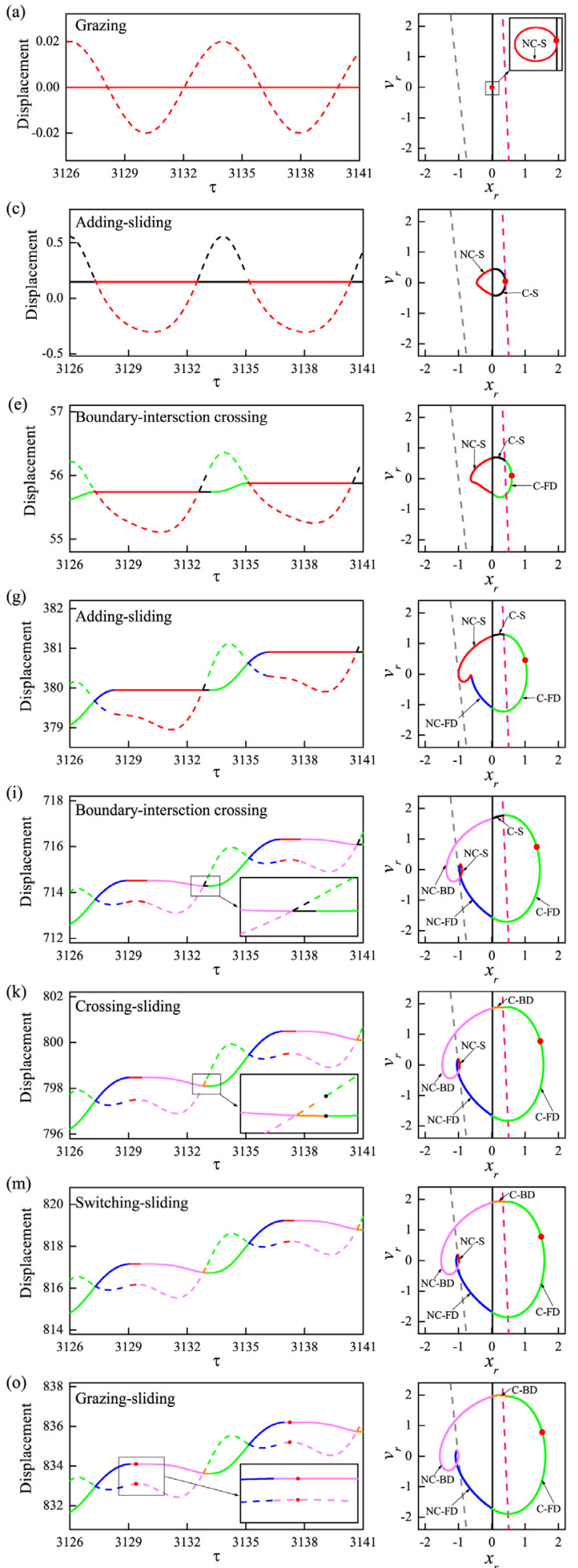
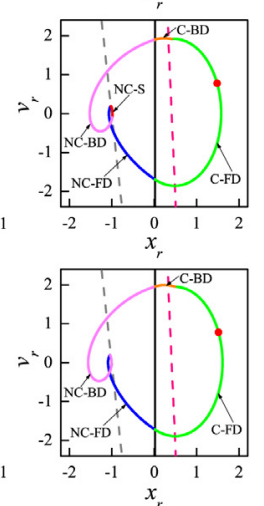

(b)
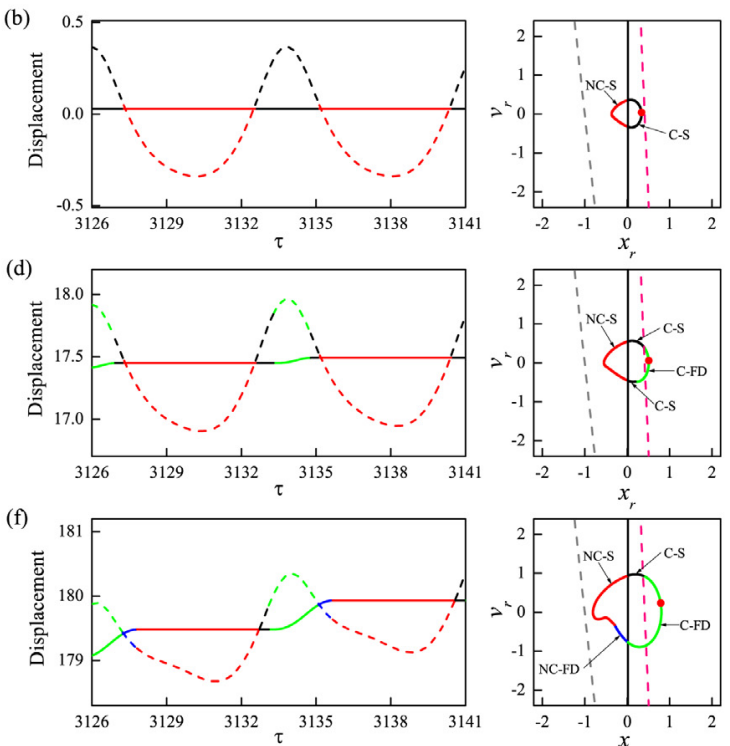

(h)
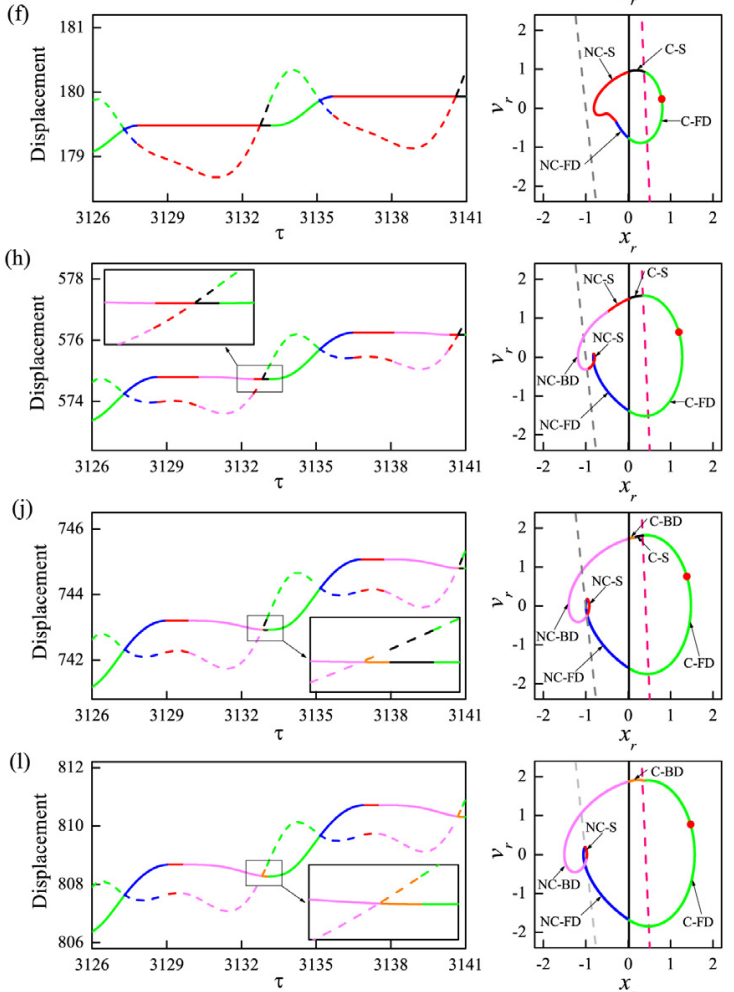

(n)
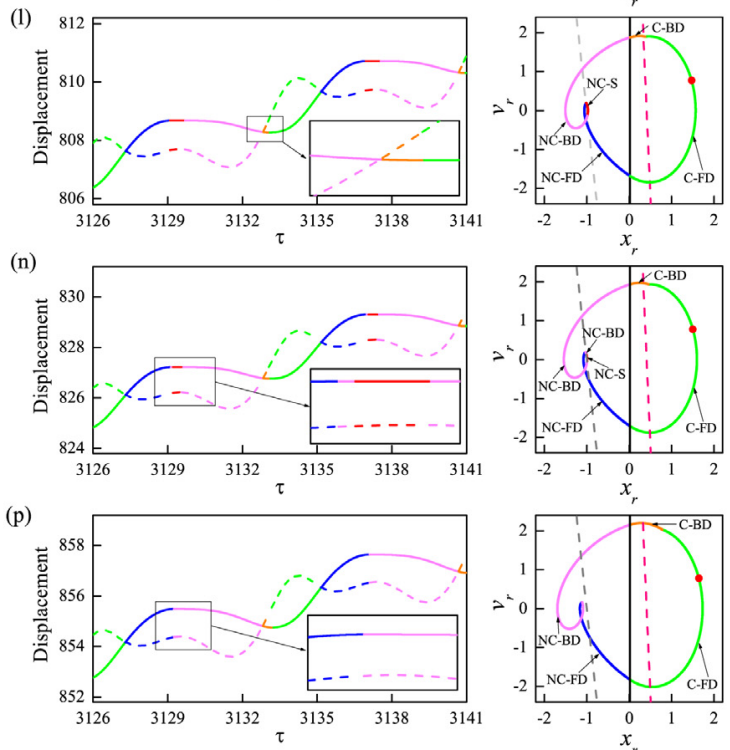

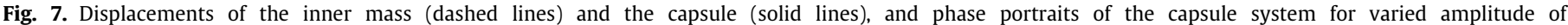

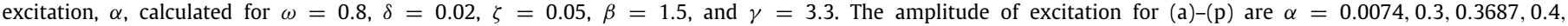

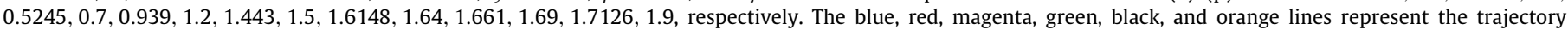

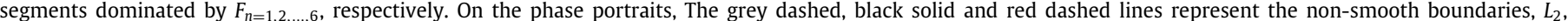
$L_{6} \cup L_{7}$ and $L_{3}$, respectively. (For interpretation of the references to colour in this figure legend, the reader is referred to the web version of this article.)

$P=P_{\Sigma_{5}, F_{3}, \Sigma_{3}} \circ P_{\Sigma_{3}, F_{2}, \Sigma_{2}} \circ P_{\Sigma_{2}, F_{1}, \Sigma_{8}} \circ P_{\Sigma_{8}, F_{4}, \Sigma_{4}} \circ P_{\Sigma_{4}, F_{5}, \Sigma_{5}}$ As the amplitude of excitation increases, the trajectory will enter the impact boundary through $S_{9}$.
- $\alpha \in(1.443,1.6148]$ : The global mapping for this regime can be represented by $P=P_{\Sigma_{5}, F_{6}, \Sigma_{9}} \circ P_{\Sigma_{9}, F_{3}, \Sigma_{3}} \circ P_{\Sigma_{3}, F_{2}, \Sigma_{2}} \circ$ $P_{\Sigma_{2}, F_{1}, \Sigma_{8}} \circ P_{\Sigma_{8}, F_{4}, \Sigma_{4}} \circ P_{\Sigma_{4}, F_{5}, \Sigma_{5}}$. As can be seen from Fig. $7(\mathrm{j})$, 
the trajectory enters the impact boundary through $S_{9}$, and the orange segment of $F_{6}(x, \tau)$ is inserted. For $\alpha=1.6148$ as shown in Fig. 7(k), a crossing-sliding bifurcation is encountered, and the trajectory approaches to $S_{4}$ after passing $L_{3}$ (There is only one point on $L_{3}$, represented by the black dot in Fig. 7(k)). Further increase of $\alpha$ will drive the trajectory from $\Sigma_{9}$ to $\Sigma_{4}$, and consequently, the sticking segment governed by $F_{5}(x, \tau)$ will vanish. Detailed 3D trajectories around $\alpha=1.6148$ are shown in Fig. 9(b).

- $\alpha \in(1.6148,1.661]$ : As shown in Fig. $7(1)$, the global mapping for this regime is given as $P=P_{\Sigma_{9}, F_{3}, \Sigma_{3}} \circ P_{\Sigma_{3}, F_{2}, \Sigma_{2}} \circ$ $P_{\Sigma_{2}, F_{1}, \Sigma_{8}} \circ P_{\Sigma_{8}, F_{4}, \Sigma_{4}} \circ P_{\Sigma_{4}, F_{6}, \Sigma_{9}}$. For $\alpha=1.661$ shown in Fig. $7(\mathrm{~m})$, a switching-sliding bifurcation occurs, and the trajectory (the blue segment) approaches to $S_{2}$ after passing $L_{2}$. If $\alpha$ is further increased, the trajectory will be driven from $S_{8}$ to $S_{3}$ rather than $S_{2}$. For this switching-sliding bifurcation, the detailed 3D trajectories around $\alpha=1.661$ are shown in Fig. 9(c).

- $\alpha \in(1.661,1.7126]$ : As illustrated in Fig. 7(n), the global mapping for this regime can be represented using $P=$ $P_{\Sigma_{9}, F_{3}, \Sigma_{3}} \circ P_{\Sigma_{3}, F_{2}, \Sigma_{2}} \circ P_{\Sigma_{2}, F_{3}, \Sigma_{3}} \circ P_{\Sigma_{3}, F_{1}, \Sigma_{8}} \circ P_{\Sigma_{8}, F_{4}, \Sigma_{4}} \circ P_{\Sigma_{4}, F_{6}, \Sigma_{9}}$. When $\alpha=1.7126$ as shown in Fig. 6(0), a grazing-sliding bifurcation is encountered, and the trajectory passes $L_{2}$ tangentially (There is only one point on $L_{2}$, represented by the red dot in Fig. 7(o)). Further increase in $\alpha$ will lead to the vanishing of sticking segment governed by $F_{2}(x, \tau)$. A detailed presentation of this grazing-sliding bifurcation around $\alpha=1.7126$ is shown in Fig. 9(d).

- $\alpha \in(1.7126,2]$ : As shown in Fig. 7(p), the global mapping for this regime can be represented using $P=P_{\Sigma_{9}, F_{3}, \Sigma_{3}}$ ○ $P_{\Sigma_{3}, F_{1}, \Sigma_{8}} \circ P_{\Sigma_{8}, F_{4}, \Sigma_{4}} \circ P_{\Sigma_{4}, F_{6}, \Sigma_{9}}$, and there is no sticking segment found in this mapping.

To depict the boundary-intersection crossing bifurcations around $\alpha=0.5245$ and $\alpha=1.443$ in detail, system trajectories are plotted with the non-smooth boundary lines in 3D space in Fig. 8. As shown in Fig. 8(a) and (b), the local mapping $P_{\Sigma_{2}, F_{4}, \Sigma_{4}}$ (the green segment) hits $L_{7}$ at $\alpha=0.5245$, which is the intersection line of $S_{5}$ and $S_{8}$. A slight increase $(\alpha=0.6)$ or decrease $(\alpha=0.4)$ in $\alpha$ will lead the trajectories to reach $S_{8}$ or $S_{5}$, respectively. As a consequence, the local and global mappings are changed, and boundary-intersection crossing bifurcation occurs around $\alpha=0.5245$. As shown in Fig. 8(c)-(d), a boundaryintersection crossing bifurcation happens around $\alpha=1.443$ and the magenta segment of $P_{\Sigma_{5}, F_{3}, \Sigma_{3}}$ hits $L_{6}$, which is the intersection line of $S_{2}$ and $S_{9}$. A slight increase $(\alpha=1.5)$ or decrease $(\alpha=1.4)$ in $\alpha$ will lead the trajectories to reach $S_{9}$ or $S_{2}$, respectively. Therefore, boundary-intersection crossing bifurcations can be detected by using the 3D local mappings, among which $P_{\Sigma_{2}, F_{4}, \Sigma_{4}}$ and $P_{\Sigma_{5}, F_{3}, \Sigma_{3}}$ indicate the occurrence of boundary-intersection crossing bifurcations on $L_{7}$ and $L_{6}$, respectively.

The non-smoothness in dry friction induces stick-slip motion for the capsule system, where sliding regions are bounded by $L_{j=1,2,3,4}$. When orbits hit on these lines, adding-sliding, crossingsliding, switching-sliding and grazing-sliding bifurcations may occur. As shown in Fig. 9, the cases for $\alpha=0.939,1.6148,1.661$, and 1.7126 are taken as examples to study each type of sliding bifurcations, which are described as follows.

- Adding-sliding bifurcation: As shown in Fig. 9(a), an adding-sliding bifurcation occurs around $\alpha=0.939$. The sliding segment $P_{\Sigma_{5}, F_{2}, \Sigma_{2}}$ is tangent to the boundary line $L_{2}^{(2)}$, and any infinitely small increase in $\alpha(\alpha=1.2)$ will lead to a backward motion segment represented by $F_{3}(x, \tau)$. Another adding-sliding bifurcation happens on $L_{3}^{(2)}$ for $\alpha=0.3687$, as shown in Fig. 6(d), and thereafter, a forward motion dominated by $F_{4}(x, \tau)$ is added. As discussed in Section 3.3, the adding-sliding bifurcation can only happen when the trajectory passes $L_{2}^{(2)}$ or $L_{3}^{(2)}$ tangentially.
- Crossing-sliding bifurcation: As shown in Fig. 9(b), a crossing-sliding bifurcation occurs around $\alpha=1.6148$, and the local mapping $P_{\Sigma_{5}, F_{6}, \Sigma_{9}}$ crosses the boundary via $L_{3}^{(3)}$. An infinitely small perturbation in $\alpha$ will change the way that the trajectory passes the non-smooth boundary of $P_{1}(x)=0$. If the trajectory passes the $P_{1}(x)=0$ plane through $S_{5}$, sliding segments dominated by $F_{5}(x, \tau)$ may exist in global mappings. If an orbit reaches $S_{4}$ directly, sliding segments dominated by $F_{5}(x, \tau)$ will disappear.

- Switching-sliding bifurcation: As shown in Fig. 9(c), a switching-sliding bifurcation occurs around $\alpha=1.661$, and the local mapping $P_{\Sigma_{2}, F_{1}, \Sigma_{8}}$ hits the boundary line $L_{2}^{(2)}$. An infinitely small perturbation in $\alpha$ will change the way how the trajectory approaches into the sliding region of $S_{2}$. For $\alpha<1.661$, e.g., $\alpha=1.64$, the segment $P_{\Sigma_{2}, F_{1}, \Sigma_{8}}$ approaches to $S_{2}$ directly. For $\alpha>1.661$, e.g., $\alpha=1.69$, the segment $P_{\Sigma_{3}, F_{1}, \Sigma_{8}}$ approaches to $S_{3}$ first and then reaches $S_{2}$.

- Grazing-sliding bifurcation: As shown in Fig. 9(d), a grazing-sliding bifurcation occurs around $\alpha=1.7126$, and the local mapping $P_{\Sigma_{2}, F_{3}, \Sigma_{3}}$ grazes the boundary line $L_{2}^{(2)}$. The segment in $\Sigma_{2}$ degenerates to the tangent point on $L_{2}^{(2)}$ and approaches to $S_{3}$ immediately. Any infinitely small increase in $\alpha$ will make the sliding segments dominated by $F_{2}(x, \tau)$ vanish.

\section{Conclusions}

This paper studied a 3D map for the piecewise-linear capsule system with bidirectional drifts. The purpose of this study is to provide a general analytical tool for studying its global and local dynamics associated with the non-smooth boundaries induced by dry friction and impact. Based on the analysis of the vector field in the vicinities of the non-smooth boundary surfaces and lines, the switching mechanisms on the non-smooth planes were determined, and all possible local mappings were obtained. By using the switching mechanisms, it can reveal the relationship between system trajectory and the non-smooth boundaries, and this could be used to explain the occurrence of boundary-intersection crossing and sliding bifurcations in the capsule system. Furthermore, the global map of the capsule system can also be obtained by constructing relevant local mappings according to the switching mechanisms studied in this paper.

By investigating various amplitudes of the external excitation, it is found that for a small amplitude ( $\alpha \leq 0.3687$ ), the capsule is kept stationary and the system orbits are constrained within the sliding regions. Adding-sliding bifurcation may occur around $\alpha=0.3687$ and $\alpha=0.939$ when system orbits are tangent to the sliding boundary lines $L_{3}^{(2)}$ and $L_{2}^{(2)}$, indicating the occurrence of forward and backward motions, respectively. Crossing-sliding, switching-sliding, and grazing-sliding bifurcations were observed when system orbits hit the sliding boundary lines $L_{j=1,2,3,4}$, determining the disappearance of sliding segments. Additionally, boundary-intersection crossing bifurcations occur when system orbits hit the intersection lines $L_{j=5,6,7,8}$, which can be detected directly by using local mappings.

Our numerical case studies demonstrate the validity of the proposed switching mechanisms on the non-smooth boundary lines and surfaces. The results illustrate that the proposed 3D global and local mappings can be used as an analytical tool to explain the occurrence of bifurcations and to investigate local and global properties of the capsule system. Future work on the 3D map will focus on deriving the analytical solution of the capsule system in order to optimise its trajectory for enhancing the rate of progression and power efficiency. 

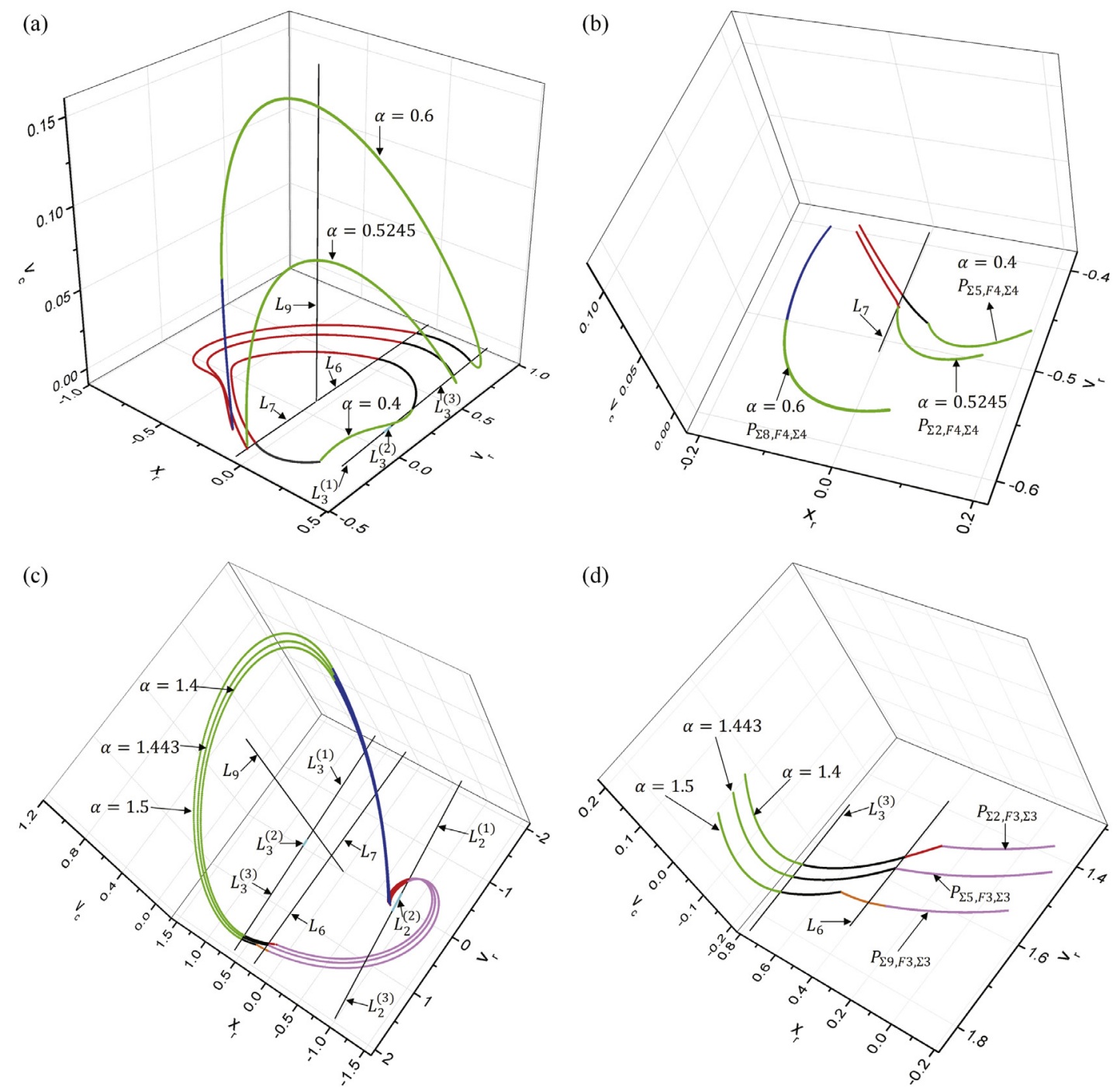

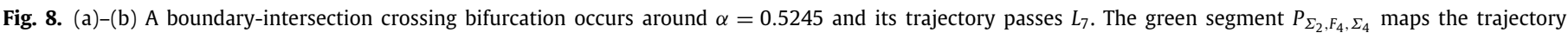
from $L_{3}^{(3)}$ to $L_{7}$ directly. For a slightly smaller $\alpha(\alpha=0.4)$, the trajectory will go out from $L_{3}^{(3)}$ and enter $S_{5}$. For a slightly larger $\alpha(\alpha=0.6)$, the segment will go out from $L_{3}^{(3)}$ and reach $S_{8}$. (c)-(d) A boundary-intersection crossing bifurcation occurs around $\alpha=1.443$ when the trajectory passes $L_{6}$. The magenta segment $P_{\Sigma_{5}, F_{3}, \Sigma_{3}}$ maps the trajectory from $L_{2}^{(1)}$ to $L_{6}$ directly. For a slightly smaller $\alpha(\alpha=1.4)$, the trajectory will go out from $L_{2}^{(1)}$ and enter $S_{2}$. For a slightly larger $\alpha(\alpha=1.5)$, the segment will go out from $L_{2}^{(1)}$ and reach $S_{9}$. The blue, red, magenta, green, black, and orange lines represent the trajectory segments dominated by $F_{n=1,2, \ldots, 6}$, respectively. (For interpretation of the references to colour in this figure legend, the reader is referred to the web version of this article.)

\section{Acknowledgements}

This work has been supported by EPSRC, UK under Grant No.

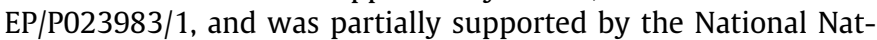
ural Science Foundation of China under Grant No. 11672257. The authors thank Professor Joseph Páez Chávez for useful discussion on constructing the 3D map.

\section{Appendix A}

The switching mechanism on $L_{1}$ depends on the sign of $\left.\left\langle\nabla P_{3}^{+}(x), F_{2}(x, \tau)\right\rangle\right|_{x=x_{1}}$, expressed in Eq. (22). Here we assume that the sign is positive for any point $x_{1}$ on $L_{1}$ at any time $\tau$, given as

$$
\left.\left\langle\nabla P_{3}^{+}(x), F_{2}(x, \tau)\right)\right|_{x=x_{1}}=v_{r}-2 \zeta f_{b}+2 \zeta \alpha \cos (\omega \tau)>0 .
$$

Eq. (A.1) can be modified as

$\alpha \cos (\omega \tau)>f_{b}-\frac{v_{r}}{2 \zeta}$.
As $x_{1}$ is a point on the line $L_{1}$, we have $v_{r}>\frac{f_{b}-\delta}{2 \zeta}$. To make Eq. (A.1) valid for any point $x_{1}$ on $L_{1}$ at any time $\tau$, one sufficient condition is

$\min (\alpha \cos (\omega \tau))>\max \left(f_{b}-\frac{v_{r}}{2 \zeta}\right)$.

Therefore, we have

$-\alpha>f_{b}-\frac{f_{b}-\delta}{4 \zeta^{2}}$

When the amplitude of the driven force $\alpha \in\left(0, \frac{f_{b}-\delta}{4 \zeta^{2}}-f_{b}\right)$, the sign of $\left.\left\langle\nabla P_{3}^{+}(x), F_{2}(x, \tau)\right\rangle\right|_{x=x_{1}}$ is positive for any point $x_{1}$ on $L_{1}$ at any time $\tau$, and hence the switching mechanism on $L_{1}$ is uni-directional, pointing from $S_{2}$ to $S_{1}$.

Based on the same principle, when the amplitude of the forcing, $\alpha \in\left(0, \frac{(1+\beta)\left(f_{b}+\delta\right)}{4 \zeta^{2}}-f_{b}\right)$, the sign of $\left.\left\langle\nabla P_{4}^{-}(x), F_{5}(x, \tau)\right)\right|_{x=x_{4}}$ is positive for any point $x_{4}$ on $L_{4}$ at any time $\tau$, and hence, the switching mechanism on $L_{4}$ is uni-directional, pointing from $S_{5}$ 

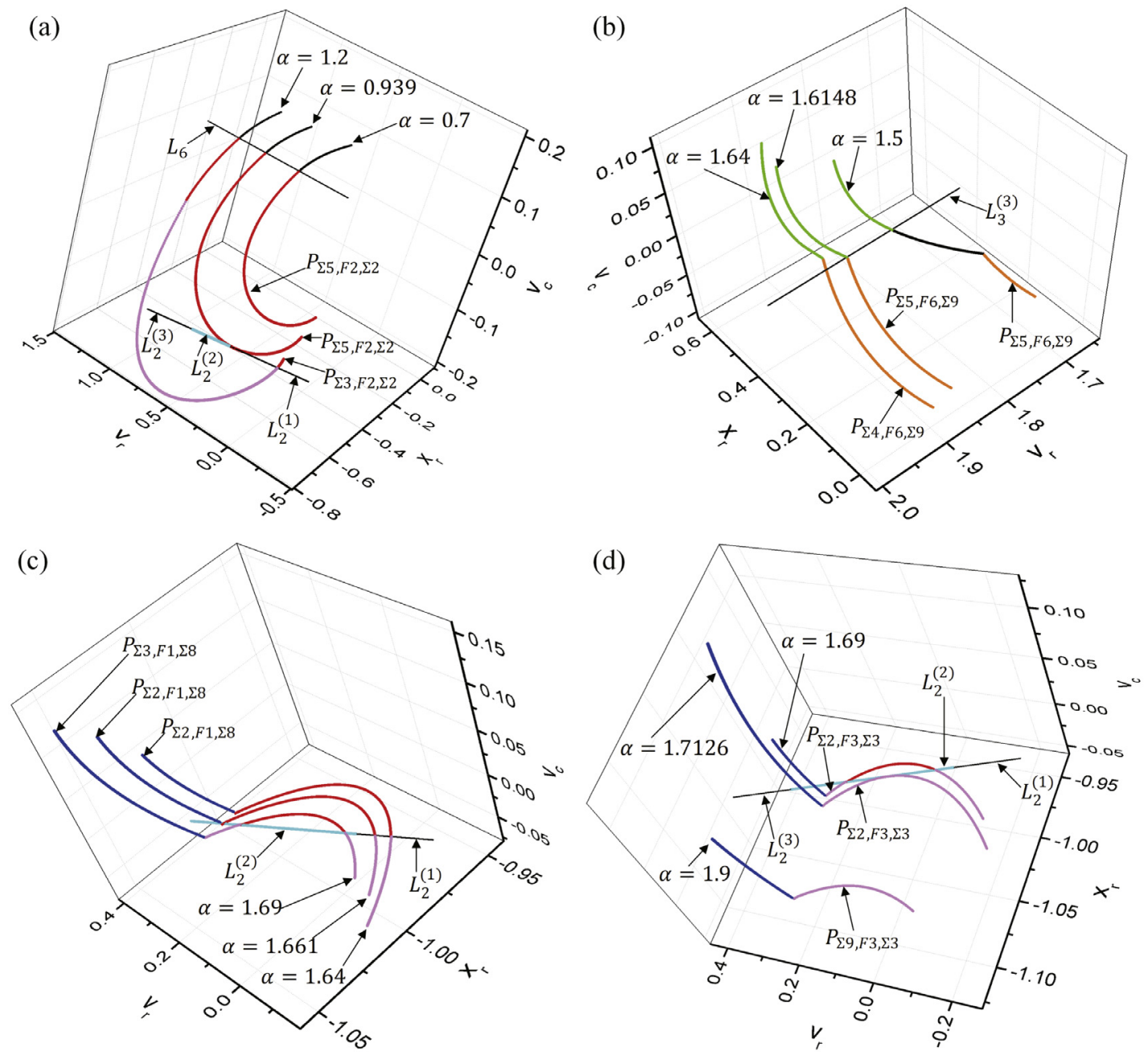

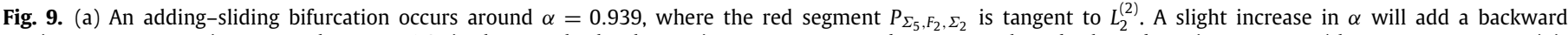

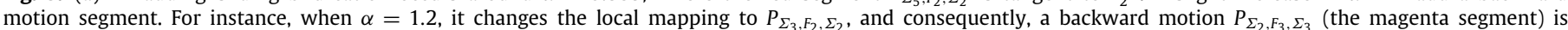

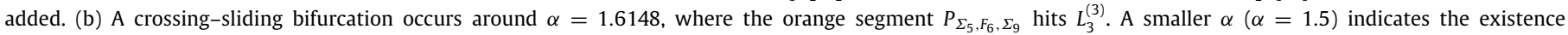

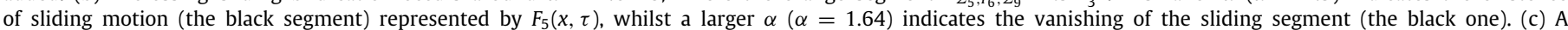

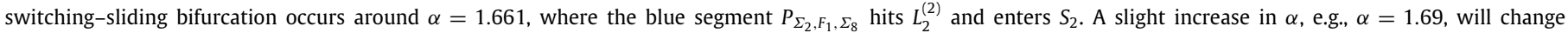

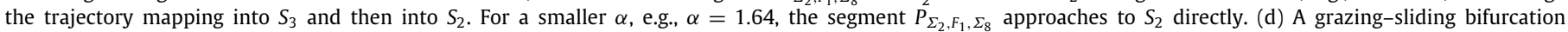

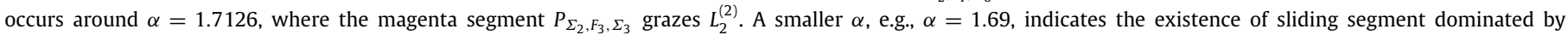

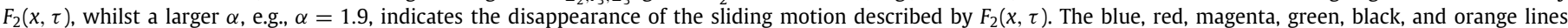

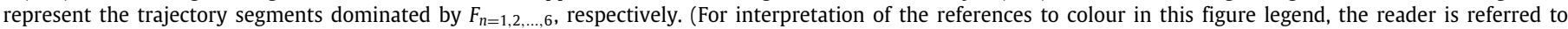
the web version of this article.)

to $S_{6}$. Therefore, $\alpha \in\left(0, \frac{f_{b}-\delta}{4 \zeta^{2}}-f_{b}\right) \cap\left(0, \frac{(1+\beta)\left(f_{b}+\delta\right)}{4 \zeta^{2}}-f_{b}\right)$ ensures that the switching directions on $L_{1}$ and $L_{4}$ are unitary.

\section{Appendix B}

The switching mechanism on the line $L_{2}$ depends on the sign of $\left.\left\langle\nabla P_{3}^{-}(x), F_{2}(x, \tau)\right)\right|_{x=x_{2}}$. To determine its sign, here we discuss the existence condition of the solution for $\left.\left\langle\nabla P_{3}^{-}(x), F_{2}(x, \tau)\right)\right|_{x=x_{2}}=0$. Here, we assume there exists $v_{r}$ and $\tau$, satisfying

$$
\left.\left\langle\nabla P_{3}^{-}(x), F_{2}(x, \tau)\right)\right|_{x=x_{2}}=-v_{r}+2 \zeta f_{b}+2 \zeta \alpha \cos (\omega \tau)=0 .
$$

Then we have

$$
-2 \zeta \alpha \leq-v_{r}+2 \zeta f_{b} \leq 2 \zeta \alpha
$$

Therefore, for a given $\alpha$, the necessary condition for the existence of the solution of Eq. (B.1) is $2 \zeta f_{b}-2 \zeta \alpha \leq v_{r} \leq 2 \zeta f_{b}+2 \zeta \alpha$. As a consequence, we will always have (i) for $v_{r}<2 \zeta f_{b}-2 \zeta \alpha$, $\left.\left\langle\nabla P_{3}^{-}(x), F_{2}(x, \tau)\right)\right|_{x=x_{2}}<0$; (ii) for $2 \zeta f_{b}-2 \zeta \alpha \leq v_{r} \leq 2 \zeta f_{b}+2 \zeta \alpha$, the sign of $\left.\left\langle\nabla P_{3}^{-}(x), F_{2}(x, \tau)\right\rangle\right|_{x=x_{2}}$ depends on the time $\tau$ significantly; and (iii) for $v_{r}>2 \zeta f_{b}+2 \zeta \alpha,\left.\left\langle\nabla P_{3}^{-}(x), F_{2}(x, \tau)\right)\right|_{x=x_{2}}>$ 0 . Therefore, the line $L_{2}$ can be divided into three segments for discussing its switching mechanism, namely $L_{2}^{(1)}$ for $v_{r} \in$ $\left[-\frac{f_{b}-\delta}{2 \zeta}, 2 \zeta f_{b}-2 \zeta \alpha\right), L_{2}^{(2)}$ for $v_{r} \in\left[2 \zeta f_{b}-2 \zeta \alpha, 2 \zeta f_{b}+2 \zeta \alpha\right]$, and $L_{2}^{(3)}$ for $v_{r} \in\left(2 \zeta f_{b}+2 \zeta \alpha,+\infty\right)$.

Based on the same principle, for a given $\alpha, L_{3}$ can be divided into three segments to discuss its switching mechanism, namely $L_{3}^{(1)}$ for $v_{r} \in\left(-\infty, \frac{2 \zeta\left(f_{b}-\alpha\right)}{1+\beta}\right), L_{3}^{(2)}$ for $v_{r} \in\left[\frac{2 \zeta\left(f_{b}-\alpha\right)}{1+\beta}, \frac{2 \zeta\left(f_{b}+\alpha\right)}{1+\beta}\right]$, and $L_{3}^{(3)}$ for $v_{r} \in\left(\frac{2 \zeta\left(f_{b}+\alpha\right)}{1+\beta}, \frac{f_{b}-\delta}{2 \zeta}\right]$.

\section{References}

[1] J.M. Mirats Tur, W. Garthwaite, Robotic devices for water main in-pipe inspection: A survey, J. Field Robotics 27 (4) (2010) 491-508. 
[2] J. Aguilar, T. Zhang, F. Qian, M. Kingsbury, B. McInroe, N. Mazouchova, C. Li, R. Maladen, C. Gong, M. Travers, et al., A review on locomotion robophysics: the study of movement at the intersection of robotics, soft matter and dynamical systems, Rep. Progr. Phys. 79 (11) (2016) 110001.

[3] B.J. Nelson, I.K. Kaliakatsos, J.J. Abbott, Microrobots for minimally invasive medicine, Annu. Rev. Biomed. Eng. 12 (2010) 55-85.

[4] F.L. Chernous'ko, On the motion of a body containing a movable internal mass, Dokl. Phys. 50 (11) (2005) 593-597.

[5] P. Valdastri, R.J. Webster III, C. Quaglia, M. Quirini, A. Menciassi, P. Dario, A new mechanism for mesoscale legged locomotion in compliant tubular environments, IEEE Trans. Robot. 25 (5) (2009) 1047-1057.

[6] F.L. Chernous'ko, The optimal periodic motions of a two-mass system in a resistant medium, J. Appl. Math. Mech. 72 (2) (2008) 116-125.

[7] E. Pavlovskaia, M. Wiercigroch, C. Grebogi, Modeling of an impact system with a drift, Phys. Rev. E 64 (5) (2001) 056224.

[8] Y. Liu, M. Wiercigroch, E. Pavlovskaia, H. Yu, Modelling of a vibro-impact capsule system, Int. J. Mech. Sci. 66 (2013) 2-11.

[9] Y. Liu, E. Pavlovskaia, M. Wiercigroch, Z. Peng, Forward and backward motion control of a vibro-impact capsule system, Int. J. Non-Linear Mech. 70 (2015) 30-46.

[10] Y. Liu, E. Pavlovskaia, M. Wiercigroch, Experimental verification of the vibro-impact capsule model, Nonlinear Dynam. 83 (1-2) (2016) 1029-1041.
[11] Y. Liu, E. Pavlovskaia, D. Hendry, M. Wiercigroch, Vibro-impact responses of capsule system with various friction models, Int. J. Mech. Sci. 72 (2013) 39-54.

[12] S.W. Shaw, P.J. Holmes, A periodically forced piecewise linear oscillator, J. Sound Vib. 90 (1) (1983) 129-155.

[13] A.B. Nordmark, Non-periodic motion caused by grazing incidence in an impact oscillator, J. Sound Vib. 145 (2) (1991) 279-297.

[14] R.P.S. Han, A.C.J. Luo, W. Deng, Chaotic motion of a horizontal impact pair, J. Sound Vib. 181 (2) (1995) 231-250.

[15] A.C.J. Luo, R.P.S. Han, The dynamics of a bouncing ball with a sinusoidally vibrating table revisited, Nonlinear Dynam. 10 (1) (1996) 1-18.

[16] M. Di Bernardo, P. Kowalczyk, A. Nordmark, Bifurcations of dynamical systems with sliding: derivation of normal-form mappings, Physica D 170 (3-4) (2002) 175-205.

[17] E. Pavlovskaia, M. Wiercigroch, Low-dimensional maps for piecewise smooth oscillators, J. Sound Vib. 305 (4-5) (2007) 750-771.

[18] G.W. Luo, X.H. Lv, L. Ma, Periodic-impact motions and bifurcations in dynamics of a plastic impact oscillator with a frictional slider, Eur. J. Mech. A Solids 27 (2008) 1088-1107.

[19] J. Páez Chávez, Y. Liu, E. Pavlovskaia, M. Wiercigroch, Path-following analysis of the dynamical response of a piecewise-linear capsule system, Commun. Nonlinear Sci. Numer. Simul. 37 (2016) 102-114. 